medRxiv preprint doi: https://doi.org/10.1101/2021.06.14.21258906; this version posted July 14, 2021. The copyright holder for this preprint (which was not certified by peer review) is the author/funder, who has granted medRxiv a license to display the preprint in perpetuity.

It is made available under a CC-BY-NC-ND 4.0 International license .

\title{
Coverage and Effectiveness of mRNA COVID-19 Vaccines among Veterans
}

Authors: Yinong Young-Xu, ScD, MA, MS ${ }^{1,2}$, Caroline Korves, $\mathrm{ScD}^{1}$, Jeff Roberts, $\mathrm{MD}^{3}$, Ethan I. Powell, $\mathrm{BA}^{1}$; Gabrielle M. Zwain, BA ${ }^{1}$, and Jeremy Smith, $\mathrm{MS}^{1}$, Hector S. Izurieta, MD, MPH, PhD ${ }^{3}$

\section{Author Institutions/Affiliations:}

1. White River Junction Veterans Affairs Medical Center, White River Junction, VT

2. Geisel School of Medicine at Dartmouth, Hanover, NH

3. Office of Vaccines Research and Review, Center for Biologics Evaluation and Research, United States Food and Drug Administration, White Oak, MD

\section{Corresponding Author:}

Yinong Young-Xu

Veterans Affairs Medical Center

215 North Main Street

White River Junction, VT 05009

Yinong.Young-Xu@va.gov

Acknowledgements of research support for the study: This project was funded by the United States Food and Drug Administration through an interagency agreement with the Veterans Health Administration

Disclaimer: This article represents the author's best judgement, and should not bind or obligate the FDA, the VA, or any other institution.

Conflict of Interest Declaration: The authors, YYX, CK, EP, GZ, JS, acknowledge having received funding from Pfizer for other research projects but not this one.

Word count: $2,782 / 3,000$

Abstract word count: 250 words

Number of Tables and/or Figures: 5 Tables and Figures

Key words: COVID-19; mRNA vaccine; SARS-CoV-2; Veterans; vaccine effectiveness 
medRxiv preprint doi: https://doi.org/10.1101/2021.06.14.21258906; this version posted July 14, 2021. The copyright holder for this preprint (which was not certified by peer review) is the author/funder, who has granted medRxiv a license to display the preprint in perpetuity. It is made available under a CC-BY-NC-ND 4.0 International license .

Abstract: (285 words)

Importance: The effectiveness of mRNA vaccination in a large and diverse American population, with older age and higher co-morbidity has not been assessed.

Objective: To describe the scope of the mRNA vaccination rollout among the diverse U.S. Veterans population, and to study the mRNA COVID-19 vaccine effectiveness (VE) against infection, symptomatic disease, hospitalization, and death.

Methods: Vaccination histories were obtained from medical records to determine if patients tested for SARSCoV-2 were unvaccinated, partially vaccinated (first dose of mRNA COVID-19 vaccine), or fully vaccinated (two doses) at time of testing. First, coverage with any COVID-19 vaccination was described for all Veterans enrolled in Veterans Health Administration (VHA). Second, to evaluate VE, a matched test-negative casecontrol evaluation was conducted utilizing SARS-CoV-2 positive (cases $[\mathrm{n}=16,690])$ and SARS-CoV-2 negative (controls $[\mathrm{n}=61,610])$ tests from Veterans aged $\geq 18$ years old who routinely sought care at a VHA facility and were tested from December 14, 2020, through March 14, 2021. VE was calculated from odds ratios (ORs) with $95 \%$ confidence intervals $(\mathrm{CI})$.

Results:

By March 7, 2021, among 6,170,750 Veterans, 1,547,045 (23\%) received at least one COVID-19 vaccination. mRNA COVID-19 VE against infection, regardless of symptoms, was 94\% (95\% CI 92-95) and 58\% (95\%CI 54-62) for full and partial vaccination (vs. no vaccination), respectively. VE against infection was similar across subpopulations, and it was not significantly different from VE against symptomatic disease. VE against COVID-19-related hospitalization and death for full vs. no vaccination was 89\% (95\%CI 81-93) and 99\% (95\%CI 87-100), respectively.

Conclusions and Relevance:

The VHA's efficient and equitable distribution of effective vaccines decreased COVID-19 infections, hospitalization, and mortality similarly for all Veterans, including Veterans with low income, homeless Veterans, immunocompromised, the elderly, minorities, and rural Veterans thus reducing health inequalities. 
medRxiv preprint doi: https://doi.org/10.1101/2021.06.14.21258906; this version posted July 14, 2021. The copyright holder for this preprint (which was not certified by peer review) is the author/funder, who has granted medRxiv a license to display the preprint in perpetuity. It is made available under a CC-BY-NC-ND 4.0 International license .

\section{INTRODUCTION}

On December 11, 2020, the United States (U.S.) Food and Drug Administration (FDA) issued an Emergency

Use Authorization (EUA) for Pfizer-BioNTech COVID-19 Vaccine, for the prevention of coronavirus disease 2019 (COVID-19) for individuals 16 years and older(1). One week later, the FDA issued an EUA for Moderna COVID-19 Vaccine, developed by Moderna, for individuals 18 years and older(2); both vaccines are mRNA vaccines. Prior to these EUAs, the pandemic's impact on Veterans enrolled in the Veterans Health Administration (VHA), like elsewhere in the US, was devastating. Approximately 207,000 COVID-19 cases had been reported by the end of 2020 among VHA enrolled Veterans(3). Additionally, the burden of COVID-19 cases was distributed disproportionally among racial and ethnic minorities and could not be explained by underlying chronic conditions. About $35 \%$ and $13 \%$ of infected Veterans were Black and Hispanic, although they comprised only $13 \%$ and $7 \%$ of the total VHA patient population, respectively. Over 10,000 of these patients died(3).

The VHA has worked closely with the Centers for Disease Control and Prevention (CDC) and other federal partners to provide COVID-19 vaccines to Veterans as quickly and safely as possible. On March 24, 2021 the success of the VHA vaccination program prompted the U.S. Congress to pass the "Strengthening and Amplifying Vaccination Efforts to Locally Immunize All Veterans and Every Spouse Act" which authorized the Department of Veterans Affairs (VA) to expand its vaccination effort beyond enrolled Veterans(4).

Rapid deployment of the vaccination program was accompanied by VHA's nationwide SARS-CoV-2 testing effort, which aimed to test and record both symptomatic and asymptomatic patients across all VHA facilities. Combined with accurate and timely recording of vaccination, this created an opportunity for a robust and wellpowered test-negative case-control analysis.

Aiming to describe the extent of vaccination coverage and evaluate the effectiveness of both mRNA vaccines in a diverse population that included socio-economically disadvantaged and medically high-risk individuals, we focused this study on the first three months of the vaccination effort in the VHA, thus the analysis excluded data on the Janssen COVID-19 Vaccine.

\section{METHODS}


medRxiv preprint doi: https://doi.org/10.1101/2021.06.14.21258906; this version posted July 14, 2021. The copyright holder for this preprint (which was not certified by peer review) is the author/funder, who has granted medRxiv a license to display the preprint in perpetuity. It is made available under a CC-BY-NC-ND 4.0 International license.

The study protocol was approved by the institutional review board of the VA Medical Center in White River Junction, VT.

\section{Data Source}

The VHA is the largest integrated health care system in the U.S., providing comprehensive care to over nine million Veterans at more than 171 medical centers and 1,112 outpatient sites of care(4). We analyzed electronic medical record data from the VHA Corporate Data Warehouse (CDW). The CDW contains patient-level information on all patient encounters, treatments, prescriptions (including vaccinations), and laboratory results rendered in VHA medical facilities.

\section{Study Design}

First, vaccination coverage, defined as having at least one COVID-19 vaccination administered at a VHA facility between December 14, 2020 and March 7, 2021, was described for the population of VHA enrollees. Second, we conducted a test-negative design (TND) case-control study to evaluate mRNA COVID-19 VE against infection, irrespective of symptoms, and a case-control study to evaluate VE against COVID-19-related hospitalization and death. The study population included Veterans ages 18 years and older, with residence in a U.S. state or Washington, D.C., who presented for SARS-CoV-2 PCR or antigen testing at a VHA outpatient or emergency room facility, or had testing within one day of hospitalization, during the study period (December 14, 2020, and March 7, 2021). Patients were required to have had VHA enrollment for at least two years prior to the study period and at least one inpatient or two outpatient visits in the past two years. Individuals meeting any of the following criteria were excluded: a COVID-19diagnosis and/or a positive SARS-CoV-2 antigen or PCR test at any time between February 2020 and study initiation (December 14, 2020); hospitalization more than one day prior to testing; incomplete VHA medical records.

For the TND case-control study to assess VE against infection, positive SARS-CoV-2 tests from qualifying patients were classified as cases. Negative SARS-CoV-2 tests from qualifying patients served as controls, and a maximum of four controls were matched to each case based on Health and Human Services (HHS) geographic region, and testing date (within 14 days of case testing date) as both of these factors are related to local disease 
medRxiv preprint doi: https://doi.org/10.1101/2021.06.14.21258906; this version posted July 14, 2021. The copyright holder for this preprint (which was not certified by peer review) is the author/funder, who has granted medRxiv a license to display the preprint in perpetuity.

It is made available under a CC-BY-NC-ND 4.0 International license .

burden, likelihood of testing positive for SARS-CoV-2, and vaccine exposure status. A TND case-control study to assess VE against symptomatic disease was also conducted. Within the population of patients with a SARSCoV-2 test performed, a case-control study was also conducted to assess VE in preventing COVID-19-related hospitalization and death. For these analyses, cases were those who tested positive and were hospitalized (or died within 30 days of testing positive), and controls were Veterans who were tested negative and did not have the outcome of interest. Up to four controls were matched to each case based on geographic region and testing date.

\section{Exposure, Outcome and Covariate Assessment}

Vaccination status was based on records of mRNA COVID-19 vaccination at a VHA facility from December 14, 2020- February 28, 2021. A person was classified as unvaccinated until the day prior to the first vaccination, partially vaccinated from day 7 after the first vaccination until the day prior to the second vaccination, and fully vaccinated starting at 7 days after the second vaccination (Supplemental Figure 1). Days 0-6 after the first and second doses were excluded from all analyses. Vaccination status was determined at the date of testing. For the TND case-control study to assess VE against infection, medical records were used to identify cases (positive tests) and controls (negative tests). For the TND case-control study to assess VE against symptomatic disease, Veterans who tested positive were further restricted to those who, on the day of testing, had evidence of at least one COVID-19 symptom (Supplemental Table 1). For the case-control study for hospitalization and death, a COVID-19-related hospitalization was identified by the presence of an admission and discharge diagnosis of COVID-19 occurring any time after the first positive SARS-CoV-2 test. A death occurring in hospital with a COVID-19 discharge diagnosis or a death occurring within 30 days of a positive SARS-CoV-2 test was classified as a COVID-19-related death; controls were drawn from patients with a negative SARSCoV-2 test who were not hospitalized for COVID-19 during the study period (or who did not die within 30 days of SARS-CoV-2 test). 
medRxiv preprint doi: https://doi.org/10.1101/2021.06.14.21258906; this version posted July 14, 2021. The copyright holder for this preprint (which was not certified by peer review) is the author/funder, who has granted medRxiv a license to display the preprint in perpetuity.

It is made available under a CC-BY-NC-ND 4.0 International license .

Demographic and clinical characteristics of patients tested for SARS-CoV-2 were assessed at the time of testing or based on data from the prior two-year period, for characteristics such as comorbidities, Charlson Comorbidity Index and Care Assessment Needs (CAN) score (See Supplemental Table 1 for full definitions).

\section{Statistical Analyses}

For VHA enrollees and for subpopulations, vaccination coverage was reported as the frequency and proportion of individuals with at least one vaccination.

For the TND case-control study, for cases (positive SARS-CoV-2 test) and controls (negative SARS-CoV-2 test), baseline demographic and clinical characteristics of the tested patients were described by reporting frequency and proportion for categorical variables and median interquartile range (IQR) for continuous variables. Missing data were reported. Standardized mean difference (SMD) was used to describe differences in characteristics between cases and controls. We used conditional logistic regression to calculate odds ratios (ORs) with $95 \%$ confidence interval (CI) for the association between positive SARS-CoV-2 testing and receipt of

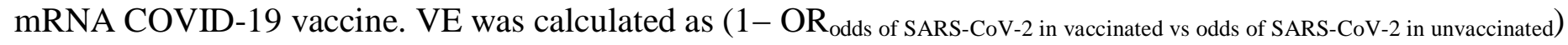
x $100 \%$. Analyses were conducted comparing partial vaccination, days 14-20 after first dose, full vaccination, and late full vaccination (day 14 after second dose and onwards) versus no vaccination. Models for adjusted analyses included covariates for potential confounders of the association between vaccination status and testing positive for SARS-CoV-2. Conditional logistic regression for the case-control analyses of hospitalization and death and for VE against symptomatic disease were conducted similarly.

All analyses were conducted using SAS, version 7. 
medRxiv preprint doi: https://doi.org/10.1101/2021.06.14.21258906; this version posted July 14, 2021. The copyright holder for this preprint (which was not certified by peer review) is the author/funder, who has granted medRxiv a license to display the preprint in perpetuity. It is made available under a CC-BY-NC-ND 4.0 International license .

\section{RESULTS}

\section{Vaccination Coverage}

By March 7, 2021, 1,547,045 (23.1\%) VHA enrollees were administered at least one dose of a COVID-19 vaccine at a VHA facility (Table 1). Vaccination coverage was higher (33.8\%) among Veterans $\geq 65$ years old. Vaccination coverage for this early vaccination period was $20.6 \%, 24.9 \%$, and $23.9 \%$ for Hispanics, Blacks and Whites, respectively. Vaccination reached homeless Veterans (19.3\%), those living in nursing homes (40.9\%) (Table 1).

\section{Study Population}

We identified 15,404 positive SARS-CoV-2 tests, classified as cases, and 497,584 negative tests, classified as controls (Figure 1). After matching, there were 15,404 cases and 61,610 controls. Baseline characteristics for cases and controls before and after matching are shown in Table 2. At the time of testing, 14,799 (96\%) cases and 50,831 (83\%) controls were unvaccinated in the matched analysis. Compared with controls, cases were younger and more likely to be White.

\section{Vaccine Effectiveness}

Table 3 shows the estimated VE for full and partial vaccination against a documented positive SARS-CoV-2 test, regardless of symptoms. Among the overall population, the adjusted VE for full and partial vaccinations were 94\% (95\%CI 92-95) and 58\% (95\%CI 54-62), respectively. VE was similar to that in the overall population for most subpopulations. VE for full vaccination was a few percentage points lower among patients who were overweight/obese (87\% (95\%CI 82-91)), had cancer (84\% (95\%CI 73-91) or had congestive heart disease $(85 \%$ (95\%CI 76-90)).

VE estimates were similar across all age, sex, race, or urban/rural status, with overlapping 95\% CIs. For the overall population and all subpopulations, the VE point estimates for partial vaccination defined by occurrence of cases within 14-20 days after the first dose were higher than those for partial vaccination measured starting 7 days after the first dose. When we restricted the analysis to symptomatic cases, VE for the overall population was 91\% (95\%CI 87-93), about the same as for all tested individuals (Supplemental Table 2). 
medRxiv preprint doi: https://doi.org/10.1101/2021.06.14.21258906; this version posted July 14, 2021. The copyright holder for this preprint (which was not certified by peer review) is the author/funder, who has granted medRxiv a license to display the preprint in perpetuity. It is made available under a CC-BY-NC-ND 4.0 International license .

Effectiveness estimates against hospitalization (89\% (95\%CI 81-93)), and against death (98.5\% (95\%CI 86.699.8), were similar to the VE we found against infection and symptomatic disease (Tables 3, 4, Supplemental Table 3). 
medRxiv preprint doi: https://doi.org/10.1101/2021.06.14.21258906; this version posted July 14, 2021. The copyright holder for this preprint (which was not certified by peer review) is the author/funder, who has granted medRxiv a license to display the preprint in perpetuity. It is made available under a CC-BY-NC-ND 4.0 International license .

\section{DISCUSSION}

The VHA's vaccination effort reached all demographic groups among Veterans in the first three months following availability of mRNA COVID vaccines under EUA. Notably, vaccination coverage was at least as high among Black Veterans as White Veterans.

This nationwide study on COVID-19 mRNA vaccines in the US demonstrated high VE of mRNA vaccines against both laboratory confirmed SARS-CoV-2 infections (regardless of symptoms) and symptomatic disease.[Table 3, Supplement Table 2] Although our sample size constrained our ability to analyze subpopulations in the short period we investigated, our VE estimates were similarly high in most subpopulations, for symptomatic cases, and against COVID-19 associated hospitalization and death. Our VE estimates are comparable to those from the clinical trials $(5,6)$ and from other observational studies

(Table 3, Supplement Table 2). Two VE studies from Israel following the rollout of Pfizer-BioNTech COVID19 Vaccine among individuals $\geq 16$ years old found similar results: a national surveillance data study, $95.3 \%$ [95\%CI 94.9-95.7\%](7), and a study in Israel's largest healthcare organization, 92\% [95\%CI 88-95\%](8). Our results were also similar to those from a prospective study among vaccinated healthcare workers in the United Kingdom, 85\% [95\%CI 74-96\%](9), and a smaller study among essential and frontline workers in the US, 90\% [95\%CI 68-97\%](10).

Although power was limited, our VE estimates against COVID-19 hospitalization were comparable to those from two studies in Israel, 97.2\% (95\%CI 96.8-97.5\%) (7) and 87\% (95\%CI 55-100\%)(8), and to a US study of patients $\geq 65$ years old, 94\% (95\%CI 49-99\%)(11) (Table 4). Also comparable were our results for VE against death, which was 96.7\% (95\%CI 96.0-97.3\%) in a study in Israel(7) (Table 4).

Our study, using data from a large and diverse U.S. population, adds important context to our understanding of VE because vaccination rollout and SARS-CoV-2 variants have differed from state to state, and country to country. In Israel and the United Kingdom, mRNA COVID-19 vaccine distribution included only the PfizerBioNTech COVID-19 Vaccine, and the United Kingdom extended the interval between doses to vaccinate their population more rapidly with one dose, limiting our ability to directly compare with their results $(8,12)$. 
medRxiv preprint doi: https://doi.org/10.1101/2021.06.14.21258906; this version posted July 14, 2021. The copyright holder for this preprint

Furthermore, each country has distinct demographic characteristics, so our study allowed for a more in-depth analysis of VE in subpopulations for whom the COVID-19 disease burden has been greater in the U.S., such as Black and Hispanic patients.

Many important prior clinical trials and observational studies $(6,7,9,10,13)$ had limited sample size to adequately assess the effectiveness of COVID-19 vaccines for people with underlying medical conditions, even though some conditions may predispose individuals to severe consequences from infection(14). The immune response to vaccination among immunocompromised individuals has not been fully explored. We found that, when fully vaccinated, effectiveness is $88 \%$ (95\%CI, 82-92) for immunocompromised patients, which is reassuring. Further evaluation of these patients by underlying disease and treatment is warranted given the response to infection may vary accordingly (14). Lee et al.(15) demonstrated COVID-19 outcomes may be more severe among patients with hematological malignancies versus solid tumors. As multiple studies, including ours, have shown $(5,9,10,12,13)$, the mRNA vaccines show some effectiveness even after just the first dose (Tables 3, 4, Supplement Table 2).

COVID-19 has disproportionally affected racial and ethnic minorities and low-income communities (16-18). Our results show the VHA was able to vaccinate minority and low-income Veterans with similar efficiency. The rates of SARS-CoV-2 testing in the first half of 2020 were higher among racial and ethnic minorities than among Whites. Prior to vaccination, African American and Hispanic Veterans were at 30 to $40 \%$ higher risk of infection compared to their Caucasian counterparts(17). After VHA's thorough effort to provide vaccination to all Veterans, regardless of racial/ethnic group or socio-economic status, we found that the risk of SARS-CoV-2 infection (irrespective of symptoms) following full vaccination was reduced for all, with similar VE for all racial and ethnic groups, demonstrating that the equitable distribution of vaccination is an effective means to reduce racial and socio-economic disparity in COVID-19 disease burden.

\section{STRENGTHS AND LIMITATIONS}

A main concern regarding test-negative studies is misclassification. Our study relied on records of vaccination collected prospectively and in near real-time rather than subjects' recall. While this study demonstrated that 
medRxiv preprint doi: https://doi.org/10.1101/2021.06.14.21258906; this version posted July 14, 2021. The copyright holder for this preprint

vaccination coverage reached all demographic subgroups, the coverage rates reported here may be conservative estimates (19), given that vaccination of individuals by State and local health departments might have not been reported to the VHA. Moreover, by the end of 2020, VHA had standardized its testing and case definitions, unlike at the beginning of the pandemic(20). We used SARS-CoV-2-positive test results coupled with COVID19 symptoms in a sensitivity analysis to reduce potential misclassification and found that VE matched that of the main analysis at 91\% (95\%CI 87, 93) (Supplemental Table 3). Some Veterans vaccinated through the VHA could have been hospitalized in a non-VHA facility, especially in rural communities (21). We limited the study population to those who routinely sought care at VHA facilities to minimize the likelihood of including patients who were vaccinated or sought treatment for COVID-19 elsewhere. To further address this, we utilized available Medicare data and repeated the analysis including any records of vaccination and hospitalization outside the VHA for these patients. The results were similar with a VE of 87\% (95\% CI 83, 89) (Supplemental Table 4). We also considered that our analysis utilized data from both antigen and PCR tests, and there may be differences in the sensitivities and specificities between tests which could lead to misclassification of cases and controls. We determined that $94 \%$ of tests were PCR and $6 \%$ were antigen tests. While this misclassification would likely be non-differential, and we repeated the analysis of VE against infection, stratifying by type of test and found no effect on the estimate (Supplemental Table 6). Our analysis included test results from all adult patients, without imposing strict rules to identify vaccine eligibility at the time of their test because the prevalence of conditions which would have made patients vaccine eligible early on was quite high (e.g., $55 \%$ hypertension among controls); we also conducted analyses for subgroups of patients who would have been vaccine eligible early on. Nevertheless, there could still be residual misclassification and differences in health seeking behavior and disease risk between different subpopulations and for individuals in the VHA and CMS system that would require further investigation in future studies.

\section{CONCLUSION}

Over a period of only three months after the first COVID-19 vaccine was authorized, the VHA successfully vaccinated and tested millions of Veterans of all socio-economic groups for COVID-19. We found mRNA 
medRxiv preprint doi: https://doi.org/10.1101/2021.06.14.21258906; this version posted July 14, 2021. The copyright holder for this preprint (which was not certified by peer review) is the author/funder, who has granted medRxiv a license to display the preprint in perpetuity.

It is made available under a CC-BY-NC-ND 4.0 International license .

vaccines to be highly effective against the risk of SARS-CoV-2 laboratory-confirmed infection, symptomatic

disease, hospitalization, and death. The effectiveness of these vaccines, combined with their equitable and

efficient deployment, have resulted in attenuated COVID-19 disease burden among all VHA enrolled Veterans

and specific vulnerable populations. 
medRxiv preprint doi: https://doi.org/10.1101/2021.06.14.21258906; this version posted July 14, 2021. The copyright holder for this preprint (which was not certified by peer review) is the author/funder, who has granted medRxiv a license to display the preprint in perpetuity.

It is made available under a CC-BY-NC-ND 4.0 International license .

Figure 1. SARS-CoV-2 PCR and antigen tests (December 14, 2020-March 7, 2021) meeting study criteria

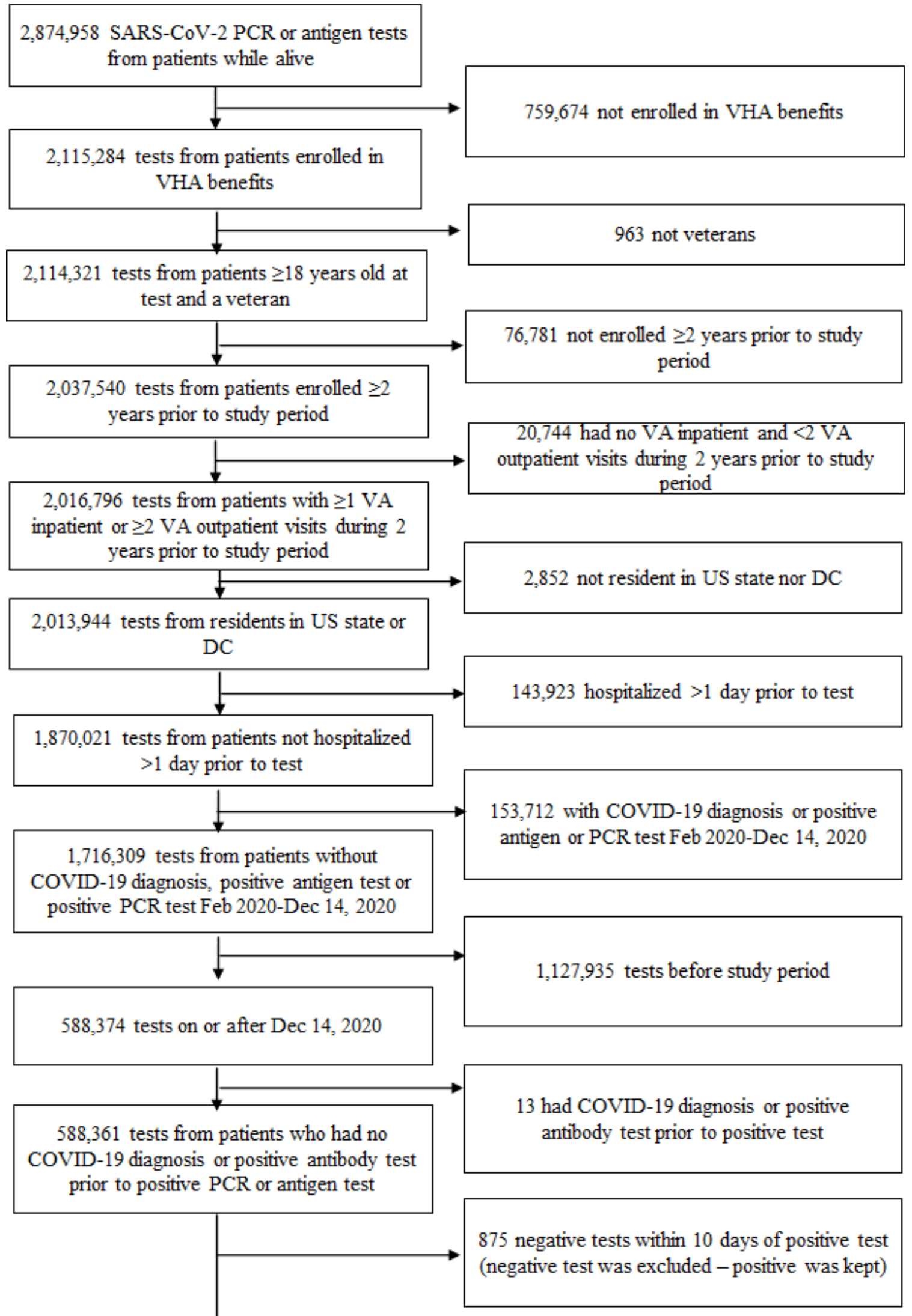


medRxiv preprint doi: https://doi.org/10.1101/2021.06.14.21258906; this version posted July 14, 2021. The copyright holder for this preprint (which was not certified by peer review) is the author/funder, who has granted medRxiv a license to display the preprint in perpetuity.

It is made available under a CC-BY-NC-ND 4.0 International license .

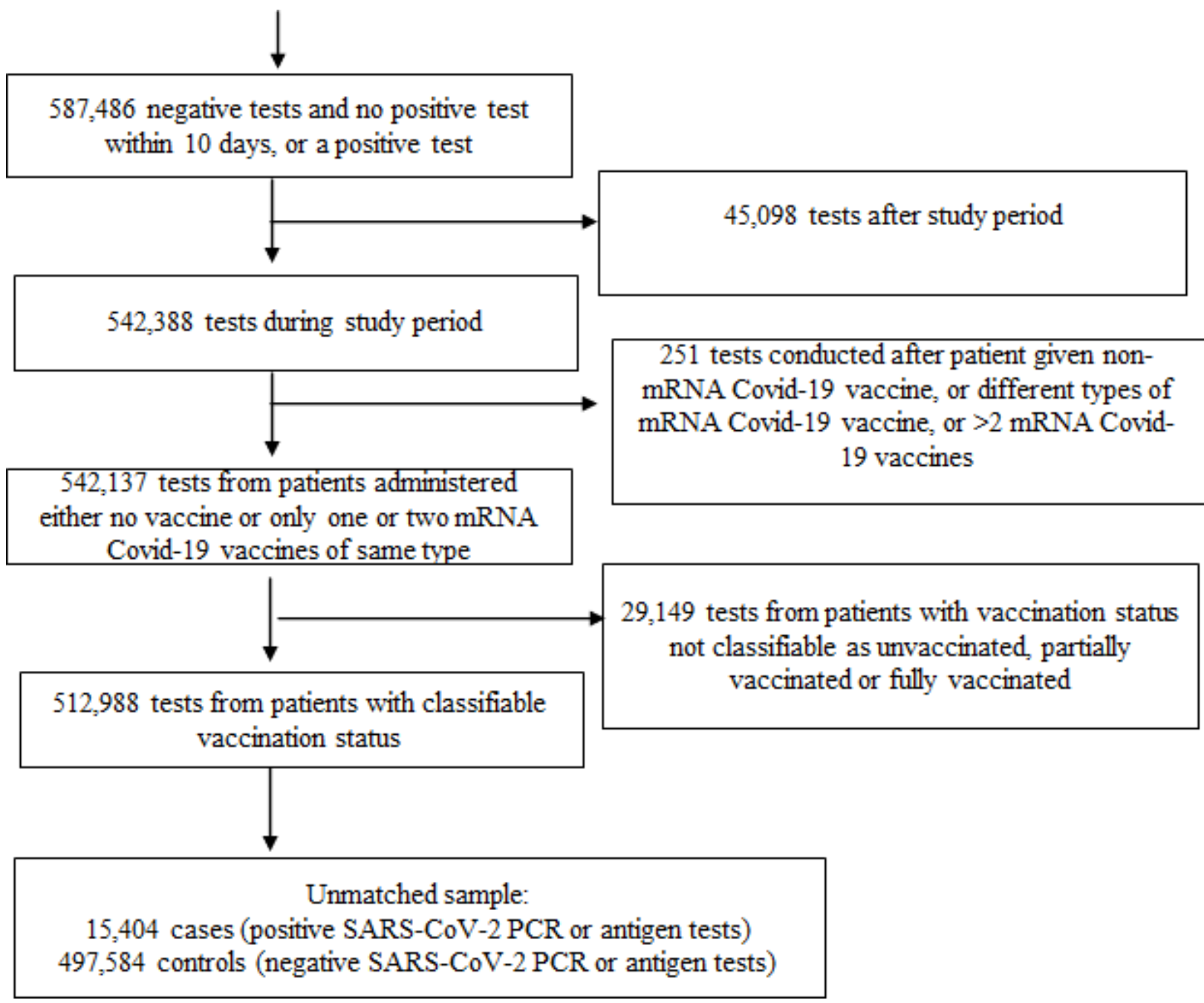


medRxiv preprint doi: https://doi.org/10.1101/2021.06.14.21258906; this version posted July 14, 2021. The copyright holder for this preprint (which was not certified by peer review) is the author/funder, who has granted medRxiv a license to display the preprint in perpetuity.

It is made available under a CC-BY-NC-ND 4.0 International license .

Table 1. COVID-19 Vaccination Coverage through March 7, 2021 among VHA Enrollees ${ }^{\mathrm{a}}$

\begin{tabular}{|c|c|c|c|}
\hline & $\begin{array}{c}\text { Number of enrollees } \\
{[\mathrm{A}]}\end{array}$ & $\begin{array}{l}\text { Vaccinated enrollees } \\
{[\mathrm{B}]}\end{array}$ & $\begin{array}{c}\% \text { Vaccination coverage } \\
{[\mathrm{B} / \mathrm{A}]}\end{array}$ \\
\hline All enrollees & $6,710,750$ & $1,547,045$ & $23 \%$ \\
\hline \multicolumn{4}{|c|}{ Age range, $\mathrm{y}$} \\
\hline $18-44$ & $1,382,988$ & 63,794 & $5 \%$ \\
\hline $45-64$ & $1,950,293$ & 340,959 & $18 \%$ \\
\hline $65-74$ & $1,895,735$ & 625,177 & $33 \%$ \\
\hline $75-84$ & $1,000,503$ & 367,395 & $37 \%$ \\
\hline$\geq 85$ & 481,231 & 149,720 & $31 \%$ \\
\hline$\geq 65$ & $3,377,469$ & $1,142,292$ & $34 \%$ \\
\hline \multicolumn{4}{|c|}{ Sex } \\
\hline Female & 634,302 & 95,579 & $15 \%$ \\
\hline Male & $6,076,448$ & $1,451,466$ & $24 \%$ \\
\hline \multicolumn{4}{|c|}{ Race or ethnic group } \\
\hline Black & $1,110,223$ & 276,750 & $25 \%$ \\
\hline Hispanic & 464,284 & 95,845 & $21 \%$ \\
\hline White & $4,407,279$ & $1,051,749$ & $24 \%$ \\
\hline Other & 384,981 & 80,993 & $21 \%$ \\
\hline Missing & 343,983 & 41,708 & $12 \%$ \\
\hline \multicolumn{4}{|c|}{ Urban-rural classification } \\
\hline Highly rural & 83,386 & 13,649 & $16 \%$ \\
\hline Rural & $2,176,409$ & 437,793 & $20 \%$ \\
\hline Urban & $4,450,955$ & $1,095,603$ & $25 \%$ \\
\hline \multicolumn{4}{|c|}{ VHA defined priority group } \\
\hline $1-4$ & $2,545,311$ & 552,638 & $22 \%$ \\
\hline $5-6$ & $2,156,949$ & 475,988 & $22 \%$ \\
\hline $7-8$ & $2,008,490$ & 518,419 & $26 \%$ \\
\hline Nursing home resident & 32,063 & 13,103 & $41 \%$ \\
\hline Homeless & 124,850 & 24,103 & $19 \%$ \\
\hline Low Income & 663,649 & 138,463 & $21 \%$ \\
\hline \multicolumn{4}{|c|}{ Comorbidities } \\
\hline Asthma & 336,849 & 135,672 & $40 \%$ \\
\hline Cancer & 191,185 & 91,660 & $48 \%$ \\
\hline Cancer, metastatic & 20,768 & 10,520 & $51 \%$ \\
\hline Coronary artery disease & 367,556 & 161,922 & $44 \%$ \\
\hline Congestive heart failure & 166,994 & 76,177 & $46 \%$ \\
\hline Chronic heart disease & 212,324 & 97,836 & $46 \%$ \\
\hline Chronic obstructive pulmonary disease & 363,203 & 146,639 & $40 \%$ \\
\hline Cardiovascular disease & 114,495 & 48,279 & $42 \%$ \\
\hline Dementia & 72,101 & 26,014 & $36 \%$ \\
\hline Dyslipidemia & 745,217 & 289,270 & $39 \%$ \\
\hline Human immunodeficiency virus (HIV) & 19,422 & 7,758 & $40 \%$ \\
\hline Hypertension & $1,473,558$ & 572,278 & $39 \%$ \\
\hline Liver disease, mild & 94,328 & 36,314 & $38 \%$ \\
\hline
\end{tabular}


medRxiv preprint doi: https://doi.org/10.1101/2021.06.14.21258906; this version posted July 14, 2021. The copyright holder for this preprint (which was not certified by peer review) is the author/funder, who has granted medRxiv a license to display the preprint in perpetuity.

It is made available under a CC-BY-NC-ND 4.0 International license .

\begin{tabular}{|c|c|c|c|}
\hline Liver disease, severe & 8,066 & 3,336 & $41 \%$ \\
\hline Myocardial infarction & 36,544 & 16,591 & $45 \%$ \\
\hline Hemiplegia or paraplegia & 19,979 & 9,048 & $45 \%$ \\
\hline Peptic ulcer disease & 8,683 & 3,761 & $43 \%$ \\
\hline Peripheral vascular disease & 140,128 & 65,357 & $47 \%$ \\
\hline Rheumatoid arthritis & 43,543 & 17,674 & $41 \%$ \\
\hline Renal disease & 219,051 & 100,879 & $46 \%$ \\
\hline Immunocompromised & 455,601 & 206,098 & $45 \%$ \\
\hline Diabetes mellitus & 967,034 & 384,596 & $40 \%$ \\
\hline
\end{tabular}


medRxiv preprint doi: https://doi.org/10.1101/2021.06.14.21258906; this version posted July 14, 2021. The copyright holder for this preprint (which was not certified by peer review) is the author/funder, who has granted medRxiv a license to display the preprint in perpetuity. It is made available under a CC-BY-NC-ND 4.0 International license .

Table 2. Exposure Status and Baseline Characteristics of Study Participants ${ }^{\mathrm{a}}$

\begin{tabular}{|c|c|c|c|c|c|c|}
\hline & \multicolumn{3}{|c|}{ Unmatched } & \multicolumn{3}{|c|}{ Matched } \\
\hline & $\begin{array}{c}\text { Cases } \\
(\mathrm{n}=15,404)\end{array}$ & $\begin{array}{c}\text { Controls } \\
(\mathrm{n}=497,584)\end{array}$ & $\mathrm{SMD}^{\mathrm{b}}$ & $\begin{array}{c}\text { Cases } \\
(\mathrm{n}=15,404)\end{array}$ & $\begin{array}{c}\text { Controls } \\
(\mathrm{n}=61,610)\end{array}$ & $\mathrm{SMD}^{\mathrm{b}}$ \\
\hline \multicolumn{7}{|c|}{ Vaccination status, No. $(\%)$} \\
\hline Full & $72(1)$ & $34,979(7)$ & 35 & $72(1)$ & $4,959(8)$ & 38 \\
\hline Partial & $533(4)$ & $36,545(7)$ & 17 & $533(4)$ & $5,820(9)$ & 25 \\
\hline None & $14,799(96)$ & $426,060(86)$ & 37 & $14,799(96)$ & $50,831(83)$ & 45 \\
\hline \multicolumn{7}{|c|}{ Vaccine manufacturer, No. $(\%)$} \\
\hline Moderna & $342(2)$ & $39,007(8)$ & 26 & $342(2)$ & $6,182(10)$ & 33 \\
\hline Pfizer-BioNTech & $263(2)$ & $32,517(7)$ & 24 & $263(2)$ & $4,597(8)$ & 28 \\
\hline $\begin{array}{l}\text { None } \\
\text { (unvaccinated) }\end{array}$ & $14,799(96)$ & $426,060(86)$ & 37 & $14,799(96)$ & $50,831(83)$ & 45 \\
\hline \multicolumn{7}{|c|}{ Month of Test, No. (\%) } \\
\hline 2020-Dec. & $4,603(30)$ & $111,052(22)$ & 17 & $4,603(30)$ & $18,349(30)$ & 0 \\
\hline 2021-Jan. & $7,306(47)$ & $190,353(38)$ & 18 & $7,306(47)$ & $25,504(41)$ & 12 \\
\hline 2021-Feb. & $2,962(19)$ & $155,601(31)$ & 28 & $2,962(19)$ & $14,537(24)$ & 11 \\
\hline 2021-Mar. & $533(4)$ & $40,578(8)$ & 20 & $533(4)$ & $3,220(5)$ & 9 \\
\hline \multicolumn{7}{|c|}{ Care setting for test, No. (\%) } \\
\hline ED/At Admission & $6,959(45)$ & $119,201(24)$ & 46 & $6,959(45)$ & $15,982(26)$ & 41 \\
\hline Outpatient & $8,445(55)$ & $378,383(76)$ & 46 & $8,445(55)$ & $45,629(74)$ & 41 \\
\hline \multicolumn{7}{|c|}{ Age, years } \\
\hline $18-44$ & $2,535(17)$ & $73,236(15)$ & 5 & $2,535(17)$ & $668(1)$ & 56 \\
\hline $45-64$ & $5,409(35)$ & $177,629(36)$ & 1 & $5,409(35)$ & $22,642(37)$ & 3 \\
\hline $65-74$ & $4,757(31)$ & $160,157(32)$ & 3 & $4,757(31)$ & $24,673(40)$ & 19 \\
\hline $75-84$ & $1,928(13)$ & $64,096(13)$ & 1 & $1,928(13)$ & $9,481(15)$ & 8 \\
\hline $85+$ & $775(5)$ & $22,466(5)$ & 2 & $775(5)$ & $4,147(7)$ & 7 \\
\hline \multicolumn{7}{|c|}{ Sex, No. $(\%)$} \\
\hline Female & $1,457(10)$ & $55,591(11)$ & 6 & $1,457(10)$ & $5,868(10)$ & 0 \\
\hline Male & $13,947(91)$ & $441,993(89)$ & 6 & $13,947(91)$ & $55,742(91)$ & 0 \\
\hline \multicolumn{7}{|c|}{ Race, No. (\%) } \\
\hline Black & $3,848(25)$ & $121,718(25)$ & 1 & $3,848(25)$ & $19,807(32)$ & 16 \\
\hline Hispanic & $1,014(7)$ & $34,816(7)$ & 2 & $1,014(7)$ & $2,884(5)$ & 8 \\
\hline White & $9,620(63)$ & $303,234(61)$ & 3 & $9,620(63)$ & $34,731(56)$ & 12 \\
\hline Other & $922(6)$ & $37,816(8)$ & 6 & $922(6)$ & $4,188(7)$ & 3 \\
\hline \multicolumn{7}{|c|}{ Rurality, No. (\%) } \\
\hline Highly rural & $107(1)$ & $3,015(1)$ & 1 & $107(1)$ & $376(1)$ & 1 \\
\hline Rural & $4,667(30)$ & $120,555(24)$ & 14 & $4,667(30)$ & $14,758(24)$ & 14 \\
\hline Urban & $10,630(69)$ & $374,014(75)$ & 14 & $10,630(69)$ & $46,476(75)$ & 14 \\
\hline \multicolumn{7}{|c|}{ HHS region, No. (\%) } \\
\hline Region 1 & $803(5)$ & $23,289(5)$ & 2 & $803(5)$ & $3,212(5)$ & 0 \\
\hline Region 2 & $763(5)$ & $23,723(5)$ & 1 & $763(5)$ & $3,052(5)$ & 0 \\
\hline Region 3 & $2,286(15)$ & $48,958(10)$ & 15 & $2,286(15)$ & $9,143(15)$ & 0 \\
\hline Region 4 & $3,944(26)$ & $138,087(28)$ & 4 & $3,944(26)$ & $15,976(26)$ & 0 \\
\hline Region 5 & $1,661(11)$ & $67,333(14)$ & 8 & $1,661(11)$ & $6,644(11)$ & 0 \\
\hline Region 6 & $3,425(22)$ & $58,556(12)$ & 28 & $3,425(22)$ & $13,696(22)$ & 0 \\
\hline Region 7 & $714(5)$ & $23,441(5)$ & 0 & $714(5)$ & $2,856(5)$ & 0 \\
\hline Region 8 & $445(3)$ & $15,880(3)$ & 2 & $445(3)$ & $1,780(3)$ & 0 \\
\hline Region 9 & $976(6)$ & $80,345(16)$ & 31 & $976(6)$ & $3,904(6)$ & 0 \\
\hline Region 10 & $337(2)$ & $17,946(4)$ & 8 & $337(2)$ & $1,348(2)$ & 0 \\
\hline Homeless, No. (\%) & $620(4)$ & $32,933(7)$ & 12 & $620(4)$ & $4,646(8)$ & 15 \\
\hline Low income, No. (\%) & $2,139(14)$ & $77,418(16)$ & 5 & $2,139(14)$ & $12,059(20)$ & 15 \\
\hline
\end{tabular}


medRxiv preprint doi: https://doi.org/10.1101/2021.06.14.21258906; this version posted July $14,2021$. The copyright holder for this preprint (which was not certified by peer review) is the author/funder, who has granted medRxiv a license to display the preprint in perpetuity. It is made available under a CC-BY-NC-ND 4.0 International license .

\begin{tabular}{|c|c|c|c|c|c|c|}
\hline $\begin{array}{l}\text { Nursing home use, } \\
\text { No. }(\%)\end{array}$ & $124(1)$ & $9,212(2)$ & 9 & $124(1)$ & $2,021(3)$ & 18 \\
\hline \multicolumn{7}{|c|}{ VA priority group, No. (\%) } \\
\hline $1-4$ & $5,823(38)$ & $210,310(42)$ & 9 & $5,823(38)$ & $32,276(52)$ & 29 \\
\hline $5-6$ & $5,131(33)$ & $161,290(32)$ & 2 & $5,131(33)$ & $17,182(28)$ & 12 \\
\hline $7-8$ & $4,450(29)$ & $125,984(25)$ & 8 & $4,450(29)$ & $12,152(20)$ & 22 \\
\hline \multicolumn{7}{|c|}{ Quan's CCI } \\
\hline Median (IQR) & $0(0,2)$ & $1(0,2)$ & & $0(0,2)$ & $1(0,3)$ & \\
\hline \multicolumn{7}{|c|}{ BMI, No. $(\%)$} \\
\hline Overweight/ obese & $7,654(50)$ & $226,955(46)$ & 8 & $7,654(50)$ & $26,933(44)$ & 12 \\
\hline \multicolumn{7}{|c|}{ Comorbidities } \\
\hline Asthma & $1,726(11)$ & $68,761(14)$ & 8 & $1,726(11)$ & $11,803(19)$ & 22 \\
\hline Cancer & $992(6)$ & $40,710(8)$ & 7 & $992(6)$ & $5,923(10)$ & 12 \\
\hline Cancer (metastatic) & $148(1)$ & $7,718(2)$ & 5 & $148(1)$ & $1,181(2)$ & 8 \\
\hline $\begin{array}{l}\text { Coronary artery } \\
\text { disease }\end{array}$ & $2,018(13)$ & $68,512(14)$ & 2 & $2,018(13)$ & $11,050(18)$ & 13 \\
\hline $\begin{array}{l}\text { Congestive heart } \\
\text { failure }\end{array}$ & $1,208(8)$ & $45,941(9)$ & 5 & $1,208(8)$ & $7,857(13)$ & 16 \\
\hline $\begin{array}{l}\text { Chronic kidney } \\
\text { disease }\end{array}$ & $1,458(10)$ & $50,718(10)$ & 2 & $1,458(10)$ & $8,585(14)$ & 14 \\
\hline $\begin{array}{l}\text { Chronic obstructive } \\
\text { pulmonary disease }\end{array}$ & $1,866(12)$ & $74,799(15)$ & 9 & $1,866(12)$ & $12,812(21)$ & 24 \\
\hline $\begin{array}{l}\text { Cardiovascular } \\
\text { disease }\end{array}$ & $676(4)$ & $30,431(6)$ & 8 & $676(4)$ & $5,464(9)$ & 18 \\
\hline Dementia & $489(3)$ & $30,981(6)$ & 14 & $489(3)$ & $6,619(11)$ & 30 \\
\hline $\begin{array}{l}\text { Diabetes mellitus } \\
\text { (complicated) }\end{array}$ & $1,827(12)$ & $58,907(12)$ & 0 & $1,827(12)$ & $10,107(16)$ & 13 \\
\hline $\begin{array}{l}\text { Diabetes mellitus } \\
\text { (uncomplicated) }\end{array}$ & $2,830(18)$ & $77,950(16)$ & 7 & $2,830(18)$ & $11,647(19)$ & 1 \\
\hline Dyslipidemia & $3,574(23)$ & $122,001(25)$ & 3 & $3,574(23)$ & $18,732(30)$ & 16 \\
\hline HIV & $82(1)$ & $4,069(1)$ & 3 & $82(1)$ & $923(2)$ & 10 \\
\hline Hypertension & $6,694(44)$ & $217,124(44)$ & 0 & $6,694(44)$ & $33,962(55)$ & 23 \\
\hline Liver disease (mild) & $578(4)$ & $24,199(5)$ & 5 & $578(4)$ & $4,098(7)$ & 13 \\
\hline $\begin{array}{l}\text { Liver disease } \\
\text { (moderate/severe) }\end{array}$ & $78(1)$ & $3,781(1)$ & 3 & $78(1)$ & $584(1)$ & 5 \\
\hline $\begin{array}{l}\text { Myocardial infarction } \\
\text { (history) }\end{array}$ & $334(2)$ & $13,182(3)$ & 3 & $334(2)$ & $2,122(3)$ & 8 \\
\hline $\begin{array}{l}\text { Peripheral vascular } \\
\text { disease }\end{array}$ & $889(6)$ & $37,031(7)$ & 7 & $889(6)$ & $6,342(10)$ & 17 \\
\hline Rheumatoid arthritis & $218(1)$ & $7,181(1)$ & 0 & $218(1)$ & $1,117(2)$ & 3 \\
\hline Renal disease & $1,491(10)$ & $51,562(10)$ & 2 & $1,491(10)$ & $8,706(14)$ & 14 \\
\hline Atrial fibrillation & $1,205(8)$ & $42,972(9)$ & 3 & $1,205(8)$ & $6,621(11)$ & 10 \\
\hline Arthritis & $1,368(9)$ & $48,622(10)$ & 3 & $1,368(9)$ & $8,294(14)$ & 15 \\
\hline Bronchiectasis & $19(0)$ & $947(0)$ & 2 & $19(0)$ & $154(0)$ & 3 \\
\hline Depression & $2,254(15)$ & $86,456(17)$ & 7 & $2,254(15)$ & $12,162(20)$ & 14 \\
\hline Embolism (history) & $304(2)$ & $12,795(3)$ & 4 & $304(2)$ & $2,164(4)$ & 9 \\
\hline Falls (history) & $114(1)$ & $6,726(1)$ & 6 & $114(1)$ & $1,163(2)$ & 10 \\
\hline Hepatitis B & $28(0)$ & $702(0)$ & 1 & $28(0)$ & $158(0)$ & 2 \\
\hline Hepatitis C & $88(1)$ & $3,995(1)$ & 3 & $88(1)$ & $880(1)$ & 9 \\
\hline Hyperlipidemia & $4,628(30)$ & $151,284(30)$ & 1 & $4,628(30)$ & $23,000(37)$ & 15 \\
\hline $\begin{array}{l}\text { Interstitial lung } \\
\text { Disease }\end{array}$ & $118(1)$ & $5,790(1)$ & 4 & $118(1)$ & $965(2)$ & 7 \\
\hline Impaired mobility & $39(0)$ & $4,903(1)$ & 9 & $39(0)$ & $1,026(2)$ & 15 \\
\hline
\end{tabular}


medRxiv preprint doi: https://doi.org/10.1101/2021.06.14.21258906; this version posted July 14, 2021. The copyright holder for this preprint (which was not certified by peer review) is the author/funder, who has granted medRxiv a license to display the preprint in perpetuity. It is made available under a CC-BY-NC-ND 4.0 International license .

\begin{tabular}{|c|c|c|c|c|c|c|}
\hline $\begin{array}{l}\text { Musculoskeletal } \\
\text { disorder }\end{array}$ & $7,390(48)$ & $255,372(51)$ & 7 & $7,390(48)$ & $36,063(59)$ & 21 \\
\hline Mycoses & $777(5)$ & $37,023(7)$ & 10 & $777(5)$ & $6,979(11)$ & 23 \\
\hline Obesity & $1,710(11)$ & $54,124(11)$ & 1 & $1,710(11)$ & $7,355(12)$ & 3 \\
\hline Parkinson's & $117(1)$ & $7,521(1)$ & 7 & $117(1)$ & $1,244(2)$ & 11 \\
\hline Sickle cell disease & $11(0)$ & $387(0)$ & 0 & $11(0)$ & $87(0)$ & 2 \\
\hline $\begin{array}{l}\text { Solid organ transplant } \\
\text { recipient }\end{array}$ & $69(0)$ & $2,756(1)$ & 2 & $69(0)$ & $422(1)$ & 3 \\
\hline $\begin{array}{l}\text { Stroke / transient } \\
\text { ischemic attack }\end{array}$ & $339(2)$ & $16,671(3)$ & 7 & $339(2)$ & $3,041(5)$ & 15 \\
\hline $\begin{array}{l}\text { Urinary tract } \\
\text { infection }\end{array}$ & $371(2)$ & $19,079(4)$ & 8 & $371(2)$ & $3,594(6)$ & 17 \\
\hline Immunocompromised & $2,324(15)$ & $96,783(20)$ & 12 & $2,324(15)$ & $14,719(24)$ & 22 \\
\hline \multicolumn{7}{|c|}{ CAN Score, Mean (SD) } \\
\hline Mortality-1 year & $5.2 \%(10)$ & $5.7 \%(11)$ & 5 & $5.2 \%(10)$ & $7.6 \%(12 \%)$ & 22 \\
\hline Mortality-90 days & $1.5 \%(4)$ & $1.7 \%(4)$ & 4 & $1.5 \%(4)$ & $2.3 \%(5 \%)$ & 17 \\
\hline Event-1 year & $20.5 \%(20)$ & $20.6 \%(21)$ & 1 & $20.5 \%(20)$ & $\begin{array}{l}24.1 \% \\
(23 \%)\end{array}$ & 16 \\
\hline Event-90 days & $8.0 \%(11)$ & $8.3 \%(12)$ & 3 & $8.0 \%(11)$ & $\begin{array}{l}10.1 \% \\
(14 \%)\end{array}$ & 17 \\
\hline $\begin{array}{l}\text { Hospitalization-1 } \\
\text { year }\end{array}$ & $17.8 \%(18)$ & $18.6 \%(19)$ & 4 & $17.8 \%(18)$ & $\begin{array}{l}21.6 \% \\
(21 \%)\end{array}$ & 19 \\
\hline $\begin{array}{l}\text { Hospitalization-90 } \\
\text { days }\end{array}$ & $7.2 \%(10)$ & $7.8 \%(11)$ & 6 & $7.2 \%(10)$ & $9.5 \%(13 \%)$ & 20 \\
\hline $\begin{array}{l}\text { Any COVID-19 } \\
\text { symptoms }\end{array}$ & $10,499(68)$ & $105,464(21)$ & 107 & $10,499(68)$ & $13,067(21)$ & 107 \\
\hline $\begin{array}{l}\text { Influenza vaccination } \\
\text { (2019/20 season) }\end{array}$ & $135(1)$ & $4,521(1)$ & 0 & $135(1)$ & $753(1)$ & 3 \\
\hline $\begin{array}{l}\text { Influenza vaccination } \\
\text { (2020/21 season) }\end{array}$ & $1,570(10)$ & $54,432(11)$ & 2 & $1,570(10)$ & 8,506 (14) & 11 \\
\hline \multicolumn{7}{|c|}{$\begin{array}{l}\text { Abbreviations: BMI, body mass index; CAN Score, Care Assessment Needs score; No., number; SMD, } \\
\text { standardized mean difference; VA, Veterans Affairs. } \\
\text { a Definitions for variables are provided in Supplemental Table } 1 . \\
\text { b }\end{array}$} \\
\hline
\end{tabular}


medRxiv preprint doi: https://doi.org/10.1101/2021.06.14.21258906; this version posted July 14, 2021. The copyright holder for this preprint (which was not certified by peer review) is the author/funder, who has granted medRxiv a license to display the preprint in perpetuity. It is made available under a CC-BY-NC-ND 4.0 International license .

Table 3. Vaccine Effectiveness Against Laboratory Confirmed SARS-CoV-2 Infection ${ }^{\mathrm{a}}$

\begin{tabular}{|c|c|c|c|c|c|}
\hline & \multicolumn{5}{|c|}{$\mathrm{VE}, \%(95 \% \mathrm{CI})$} \\
\hline & \multicolumn{3}{|c|}{ Full vs. no vaccination } & \multicolumn{2}{|c|}{ Partial vs. no vaccination } \\
\hline & \multicolumn{2}{|c|}{ Unadjusted } & Adjusted $^{\mathrm{b}}$ & Unadjusted & Adjusted $^{\mathrm{b}}$ \\
\hline Overall & \multicolumn{2}{|c|}{$96(95,97)$} & $94(92,95)$ & $70(67 \%, 73 \%)$ & $58(54,62)$ \\
\hline \multicolumn{6}{|c|}{ Age, y } \\
\hline $18-64$ & \multicolumn{2}{|c|}{$91(84,95)$} & $91(83,95)$ & $59(49,67)$ & $52(40,62)$ \\
\hline$\geq 65$ & \multicolumn{2}{|c|}{$92(90,94)$} & $92(89,94)$ & $57(52,61)$ & $54(48,58)$ \\
\hline $18-79$ & \multicolumn{2}{|c|}{$91(88,93)$} & $91(88,93)$ & $55(50,59)$ & $53(48,58)$ \\
\hline$\geq 80$ & \multicolumn{2}{|c|}{$93(89,96)$} & $93(88,96)$ & $59(50,66)$ & $56(46,64)$ \\
\hline \multicolumn{6}{|c|}{ VHA defined priority group } \\
\hline $1-4$ & \multicolumn{2}{|c|}{$93(90,95)$} & $93(89,95)$ & $60(53,65)$ & $56(49,63)$ \\
\hline $5-6$ & \multicolumn{2}{|c|}{$88(83,92)$} & $90(85,93)$ & $53(45,60)$ & $55(46,62)$ \\
\hline $7-8$ & \multicolumn{2}{|c|}{$90(84,94)$} & $90(84,94)$ & $43(33,52)$ & $47(37,56)$ \\
\hline \multicolumn{6}{|c|}{ Race or ethnic group } \\
\hline Black & \multicolumn{2}{|c|}{$94(89,97)$} & $93(88,96)$ & $49(39,58)$ & $48(37,57)$ \\
\hline Hispanic & \multicolumn{2}{|c|}{$82(59,92)$} & $73(38,89)$ & $52(25,69)$ & $44(13,65)$ \\
\hline White & \multicolumn{2}{|c|}{$91(88,93)$} & $90(87,93)$ & $56(51,61)$ & $55(50,60)$ \\
\hline Other & \multicolumn{2}{|c|}{$98(86,100)$} & $98(86,100)$ & $61(40,75)$ & $65(46,78)$ \\
\hline \multicolumn{6}{|c|}{ Sex } \\
\hline Female & \multicolumn{2}{|c|}{$86(62,95)$} & $82(51,94)$ & $73(50,85)$ & $65(35,81)$ \\
\hline Male & \multicolumn{2}{|c|}{$92(90,94)$} & $92(90,94)$ & $54(49,58)$ & $54(50,58)$ \\
\hline \multicolumn{6}{|c|}{ Urban-rural } \\
\hline Rural & \multicolumn{2}{|c|}{$93(89,96)$} & $93(89,96)$ & $60(52,66)$ & $58(50,65)$ \\
\hline Urban & \multicolumn{2}{|c|}{$91(88,93)$} & $90(87,93)$ & $52(47,57)$ & $50(44,55)$ \\
\hline & & Income & & & \\
\hline Low income & $92(\varepsilon$ & $3,96)$ & $92(84,96)$ & $58(45,68)$ & $56(42,66)$ \\
\hline & & Homeless & & & \\
\hline Homeless & $93(7$ & $3,98)$ & $93(79,98)$ & $60(36,76)$ & $56(28,73)$ \\
\hline & & BMI & & & \\
\hline Overweight/Obese & $88(\varepsilon$ & $3,92)$ & $87(82,91)$ & $48(41,55)$ & $45(37,52)$ \\
\hline & & derlying medical con & itions & & \\
\hline Cancer & $85(7$ & $4,91)$ & $84(73,91)$ & $45(28,58)$ & $43(25,56)$ \\
\hline $\begin{array}{l}\text { Congestive heart } \\
\text { failure }\end{array}$ & $87(\varepsilon$ & , 92) & $85(76,90)$ & $57(46,66)$ & $54(42,64)$ \\
\hline $\begin{array}{l}\text { Chronic kidney } \\
\text { disease }\end{array}$ & $93(\varepsilon$ & $7,96)$ & $91(85,94)$ & $54(43,63)$ & $56(45,64)$ \\
\hline Diabetes mellitus & $93(9$ & , 95) & $92(88,94)$ & $59(53,65)$ & $56(49,62)$ \\
\hline Hypertension & $91(\varepsilon$ & $3,93)$ & $91(88,93)$ & $56(50,61)$ & $56(51,61)$ \\
\hline Immunocompromised & 86 & $9,90)$ & $88(82,92)$ & $42(31,52)$ & $43(32,52)$ \\
\hline & Adjusted VE, $\%$ & (95\%CI) [reference & oup: unvaccina & & \\
\hline & $\begin{array}{l}\text { Late full } \\
\text { vaccination (from } \\
14 \text { days after dose } \\
\text { 2) }\end{array}$ & $\begin{array}{l}\text { Full vaccination } \\
\text { (from } 7 \text { days after } \\
\text { dose } 2 \text { ) }\end{array}$ & $\begin{array}{l}14 \text { to } 20 \text { days } \\
\text { after dose } 1\end{array}$ & $\begin{array}{l}\text { Partial vaccinati } \\
\text { after dose } 1 \text { un }\end{array}$ & $\begin{array}{l}\text { (7 days } \\
\text { dose } 2)\end{array}$ \\
\hline & & Overall & & & \\
\hline & $95(3,96)$ & $94(92,95)$ & $63(57,69)$ & $58(54$ & 62) \\
\hline & & BMI & & & \\
\hline Overweight/Obese & $91(85,95)$ & $87(82,91)$ & $58(46,67)$ & $45(37$ & 52) \\
\hline
\end{tabular}


medRxiv preprint doi: https://doi.org/10.1101/2021.06.14.21258906; this version posted July 14, 2021. The copyright holder for this preprint (which was not certified by peer review) is the author/funder, who has granted medRxiv a license to display the preprint in perpetuity. It is made available under a CC-BY-NC-ND 4.0 International license .

\begin{tabular}{|l|c|c|c|c|}
\hline Cancer & $86(73,93)$ & $84(73,91)$ & $56(29,73)$ & $43(25,56)$ \\
\hline $\begin{array}{c}\text { Congestive heart } \\
\text { failure }\end{array}$ & $88(79,94)$ & $85(76,90)$ & $70(53,81)$ & $54(42,64)$ \\
$\begin{array}{c}\text { Chronic kidney } \\
\text { disease }\end{array}$ & $92(84,95)$ & $91(85,94)$ & $66(49,78)$ & $55(45,64)$ \\
\hline Diabetes mellitus & $93(88,95)$ & $92(88,94)$ & $64(53,72)$ & $56(49,62)$ \\
\hline Hypertension & $92(89,95)$ & $91(88,93)$ & $62(53,69)$ & $56(51,61)$ \\
\hline Immunocompromised & $86(78,91)$ & $88(82,92)$ & $57(41,68)$ & $43(32,52)$ \\
\hline
\end{tabular}

Abbreviations: BMI, body mass index; VE, vaccine effectiveness; VHA, Veterans Health Affairs.

${ }^{a}$ See Supplemental Table 1 for definitions of variables in this table.

${ }^{\mathrm{b}}$ The adjusted variables include the following: age, BMI, cancer, congestive heart failure, chronic kidney disease, diabetes mellitus, hypertension, immunocompromised, VA priority level, race, sex, rurality. 
medRxiv preprint doi: https://doi.org/10.1101/2021.06.14.21258906; this version posted July 14, 2021. The copyright holder for this preprint

(which was not certified by peer review) is the author/funder, who has granted medRxiv a license to display the preprint in perpetuity.

It is made available under a CC-BY-NC-ND 4.0 International license .

Table 4. Vaccine Effectiveness Against COVID-19-related Hospitalization and Death ${ }^{\mathrm{a}}$

\begin{tabular}{|c|c|c|c|c|}
\hline & \multicolumn{4}{|c|}{ VE against COVID-19-related hospitalization, \% (95\% CI) } \\
\hline & \multicolumn{2}{|c|}{ Full vs. no vaccination } & \multicolumn{2}{|c|}{ Partial vs. no vaccination } \\
\hline & Unadjusted & Adjusted $^{\mathrm{b}}$ & Unadjusted & Adjusted $^{\mathrm{b}}$ \\
\hline \multirow[t]{4}{*}{ Overall } & $87(78,92)$ & $89(81,93)$ & $31(17,43)$ & $40(27,50)$ \\
\hline & \multicolumn{4}{|c|}{ VE against COVID-19-related death, \% (95\% CI) } \\
\hline & \multicolumn{2}{|c|}{ Full vs. no vaccination } & \multicolumn{2}{|c|}{ Partial vs. no vaccination } \\
\hline & Unadjusted & Adjusted $^{\mathrm{b}}$ & Unadjusted & Adjusted $^{\mathrm{b}}$ \\
\hline Overall & $95(64,99)$ & $99(87,100)$ & $37(2,60)$ & $55(21,74)$ \\
\hline \multicolumn{5}{|c|}{$\begin{array}{l}\text { Abbreviations: BMI, body mass index; VE, vaccine effectiveness; VHA, Veterans Health Affairs. } \\
\text { a See Supplemental Table } 1 \text { for definitions of variables in this table. } \\
{ }^{\mathrm{b}} \text { The adjusted variables include the following: age, BMI, cancer, congestive heart failure, chronic kidney disease, diabetes } \\
\text { mellitus, hypertension, immunocompromised, VA priority level, race, sex, rurality. }\end{array}$} \\
\hline
\end{tabular}


medRxiv preprint doi: https://doi.org/10.1101/2021.06.14.21258906; this version posted July 14, 2021. The copyright holder for this preprint (which was not certified by peer review) is the author/funder, who has granted medRxiv a license to display the preprint in perpetuity. It is made available under a CC-BY-NC-ND 4.0 International license .

\section{Supplementary Material}

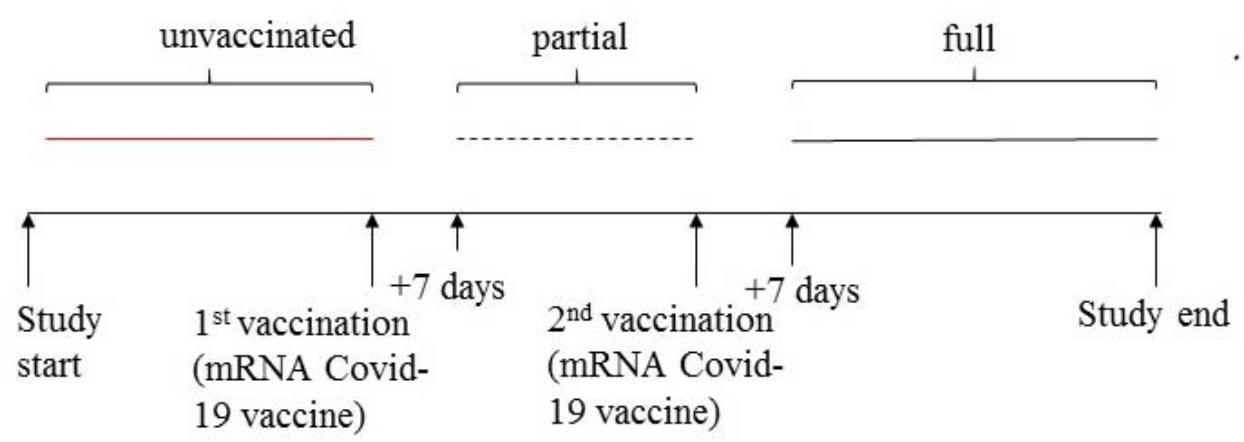

Supplemental Figure 1. Vaccination exposure classification 
medRxiv preprint doi: https://doi.org/10.1101/2021.06.14.21258906; this version posted July 14, 2021. The copyright holder for this preprint (which was not certified by peer review) is the author/funder, who has granted medRxiv a license to display the preprint in perpetuity.

It is made available under a CC-BY-NC-ND 4.0 International license.

Supplemental Table 1. Definitions of variables

\begin{tabular}{|c|c|c|c|}
\hline Variable & Values & Definition & Timing \\
\hline Age & Integer & $\begin{array}{l}\text { Age as of the specimen } \\
\text { collection date for SARS-CoV- } \\
2 \text { lab test }\end{array}$ & $\begin{array}{l}\text { Table 1: Determined at March 7, } \\
\text { 2021; Table 2: Determined at the } \\
\text { specimen collection date for } \\
\text { SARS-CoV-2 lab test during the } \\
\text { study period (December } 14,2020 \\
\text { to March 7, 2021) }\end{array}$ \\
\hline Sex & Female/male & As defined in VHA data & Most recent available \\
\hline Race & $\begin{array}{l}\text { Black } \\
\text { Hispanic } \\
\text { White } \\
\text { Other }\end{array}$ & $\begin{array}{l}\text { Black: non-Hispanic black } \\
\text { Hispanic: Hispanic any race } \\
\text { White: non-Hispanic white } \\
\text { Other: non-Hispanic other race, } \\
\text { missing race, declined to state, } \\
\text { unknown }\end{array}$ & Most recent available \\
\hline Rurality & $\begin{array}{l}\text { Highly Rural } \\
\text { Rural } \\
\text { Urban }\end{array}$ & $\begin{array}{l}\text { VHA defined based on the } \\
\text { Rural-Urban Community Area } \\
\text { (RUCA) system } \\
\text { [ https://www.ruralhealth.va.go } \\
\text { v/rural-definition.asp] }\end{array}$ & Most recent available \\
\hline $\begin{array}{l}\text { VHA defined priority } \\
\text { group }\end{array}$ & $1,2,3,4,5,6,7,8$ & $\begin{array}{l}\text { VA defined based on factors } \\
\text { including military service } \\
\text { history, disability rating and } \\
\text { income level to identify } \\
\text { Veterans to determine } \\
\text { enrolment priority; } 1 \text { is the } \\
\text { highest priority } \\
\text { [https://www.va.gov/health- } \\
\text { care/eligibility/priority-groups/] }\end{array}$ & Most recent available \\
\hline Nursing home use & $0 / 1$ & $\begin{array}{l}1=\text { Any nursing home or long- } \\
\text { term care indicated on an } \\
\text { inpatient admission or place of } \\
\text { disposition }\end{array}$ & $\begin{array}{l}\text { During } 2 \text { years prior to and on date } \\
\text { of specimen collection date for } \\
\text { SARS-CoV-2 lab test }\end{array}$ \\
\hline $\begin{array}{l}\text { Health and Human } \\
\text { Services (HHS) region }\end{array}$ & $\begin{array}{l}\text { Region } 1 \\
\text { Region } 2 \\
\text { Region } 3 \\
\text { Region } 4 \\
\text { Region } 5 \\
\text { Region } 6 \\
\text { Region } 7 \\
\text { Region } 8 \\
\text { Region } 9 \\
\text { Region } 10\end{array}$ & $\begin{array}{l}\text { Region 1: (state abbreviation) } \\
\text { CT, ME, NH, RI, VT } \\
\text { Region 2: NJ, NY } \\
\text { Region 3: DE, DC, MD, PA, } \\
\text { VA, WV } \\
\text { Region 4: AL, FL, GA, KY, } \\
\text { MS, NC, SC, TN } \\
\text { Region 5: IL, IN, MI, MN, OH, } \\
\text { WI } \\
\text { Region 6: AR, LA, NM, OK, } \\
\text { TX } \\
\text { Region 7: IA, KS, MO, NE } \\
\text { Region 8: CO, MT, ND, SD, } \\
\text { UT, WY } \\
\text { Region 9: AZ, CA, HI, NV } \\
\text { Region 10: AK, ID, OR, WA }\end{array}$ & $\begin{array}{l}\text { Most recent available value based } \\
\text { on location of patient's residence }\end{array}$ \\
\hline Homeless & $0 / 1$ & $\begin{array}{l}\text { 1= ICD-10 code Z59.0 or } \\
\text { Z59.1 } \\
\text { on any inpatient or outpatient }\end{array}$ & $\begin{array}{l}\text { During } 2 \text { years prior to specimen } \\
\text { collection date for SARS-CoV-2 } \\
\text { lab test }\end{array}$ \\
\hline
\end{tabular}


medRxiv preprint doi: https://doi.org/10.1101/2021.06.14.21258906; this version posted July 14, 2021. The copyright holder for this preprint (which was not certified by peer review) is the author/funder, who has granted medRxiv a license to display the preprint in perpetuity.

It is made available under a CC-BY-NC-ND 4.0 International license .

\begin{tabular}{|c|c|c|c|}
\hline & & record & \\
\hline Low Income & $0 / 1$ & $\begin{array}{l}1=\text { income less than } 12,760, \\
\text { but not missing income } \\
0=\text { missing or income greater } \\
\text { than equal to } 12760\end{array}$ & $\begin{array}{l}\text { During } 2 \text { years prior to specimen } \\
\text { collection date for SARS-CoV-2 } \\
\text { lab test }\end{array}$ \\
\hline Quan-CCI & Numeric & $\begin{array}{l}\text { Using ICD-10 codes from any } \\
\text { inpatient or outpatient record, } \\
\text { computed Charlson } \\
\text { Comorbidity Index, Quan's } \\
\text { version [Quan H, Sundararajan } \\
\text { V, Halfon P, Fong A, Burnand } \\
\text { B, Luthi JC, et al. Coding } \\
\text { algorithms for defining } \\
\text { comorbidities in ICD-9-CM } \\
\text { and ICD-10 administrative } \\
\text { data. Medical Care 2005; } \\
\text { 43(11):1130-1139. DOI: } \\
\text { 10.1097/01.mlr.0000182534.19 } \\
\text { 832.83] }\end{array}$ & $\begin{array}{l}\text { During } 2 \text { years prior to and on date } \\
\text { of specimen collection date for } \\
\text { SARS-CoV-2 lab test }\end{array}$ \\
\hline BMI & $\begin{array}{l}\text { Normal } \\
\text { Overweight/obese } \\
\text { Missing }\end{array}$ & $\begin{array}{l}\text { Normal: BMI less than } 26 \\
\text { Overweight/obese: BMI greater } \\
\text { than or equal to } 26\end{array}$ & $\begin{array}{l}\text { During } 2 \text { years prior to and on date } \\
\text { of specimen collection date for } \\
\text { SARS-CoV-2 lab test }\end{array}$ \\
\hline Comorbidities & & $\begin{array}{l}\text { All comorbidities listed below } \\
\text { have } 1 \text { inpatient or } 2 \text { outpatient } \\
\text { records with the corresponding } \\
\text { ICD- } 10 \text { code }\end{array}$ & $\begin{array}{l}\text { During } 2 \text { years prior to and on date } \\
\text { of specimen collection date for } \\
\text { SARS-CoV-2 lab test }\end{array}$ \\
\hline Asthma & $0 / 1$ & $\begin{array}{l}\text { ICD-10 code } \mathrm{J} 45^{*} \\
\text { ICD-10 code J44.9* } \\
\text { ICD-10 code J67.8* }\end{array}$ & \\
\hline Cancer & $0 / 1$ & $\begin{array}{l}\text { Charlson condition definitions } \\
\text { using ICD-10 codes }\end{array}$ & \\
\hline Cancer metastatic & $0 / 1$ & $\begin{array}{l}\text { Charlson condition definitions } \\
\text { using ICD-10 codes }\end{array}$ & \\
\hline Coronary artery disease & $0 / 1$ & ICD-10 code I 25* & \\
\hline Congestive heart failure & $0 / 1$ & $\begin{array}{l}\text { Charlson condition definitions } \\
\text { using ICD-10 codes }\end{array}$ & \\
\hline Chronic kidney disease & $0 / 1$ & ICD-10 code N18* & \\
\hline $\begin{array}{l}\text { Chronic obstructive } \\
\text { pulmonary disease }\end{array}$ & $0 / 1$ & $\begin{array}{l}\text { Charlson condition definitions } \\
\text { using ICD- } 10 \text { codes }\end{array}$ & \\
\hline Cardiovascular disease & $0 / 1$ & $\begin{array}{l}\text { Charlson condition definitions } \\
\text { using ICD- } 10 \text { codes }\end{array}$ & \\
\hline Dementia & $0 / 1$ & $\begin{array}{l}\text { Charlson condition definitions } \\
\text { using ICD-10 codes }\end{array}$ & \\
\hline $\begin{array}{r}\text { Diabetes mellitus with } \\
\text { complications }\end{array}$ & $0 / 1$ & $\begin{array}{l}\text { Charlson condition definitions } \\
\text { using ICD-10 codes }\end{array}$ & \\
\hline $\begin{array}{r}\text { Diabetes mellitus without } \\
\text { complications }\end{array}$ & $0 / 1$ & $\begin{array}{l}\text { Charlson condition definitions } \\
\text { using ICD- } 10 \text { codes }\end{array}$ & \\
\hline Dyslipidemia & $0 / 1$ & ICD-10 code E78.5* & \\
\hline HIV & $0 / 1$ & $\begin{array}{l}\text { Charlson condition definitions } \\
\text { using ICD- } 10 \text { codes }\end{array}$ & \\
\hline Hypertension & $0 / 1$ & $\begin{array}{l}\text { ICD-10 code } \mathrm{H} 35.03 \\
\text { ICD-10 code } 110 *\end{array}$ & \\
\hline
\end{tabular}


medRxiv preprint doi: https://doi.org/10.1101/2021.06.14.21258906; this version posted July 14, 2021. The copyright holder for this preprint (which was not certified by peer review) is the author/funder, who has granted medRxiv a license to display the preprint in perpetuity.

It is made available under a CC-BY-NC-ND 4.0 International license .

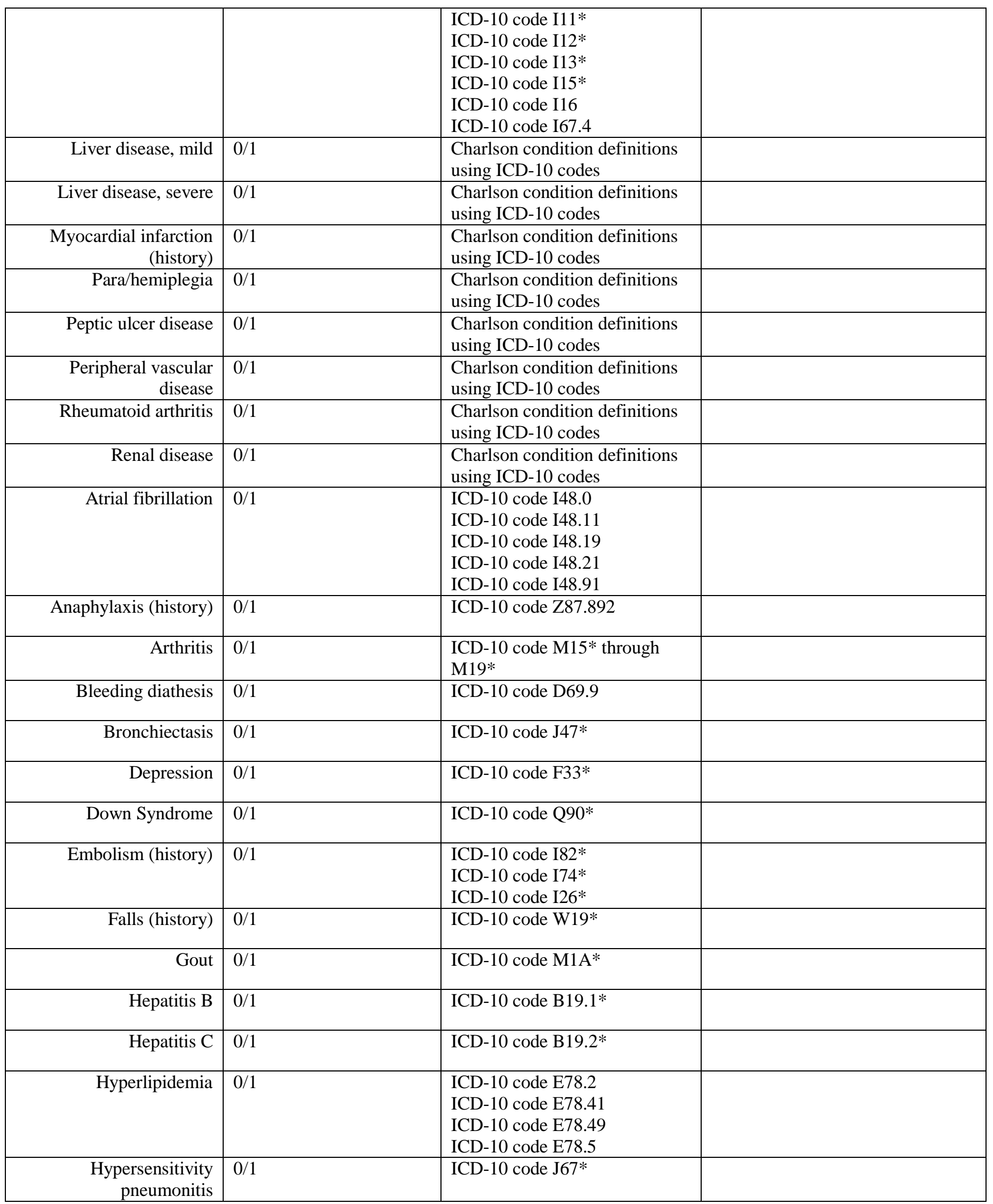


medRxiv preprint doi: https://doi.org/10.1101/2021.06.14.21258906; this version posted July 14, 2021. The copyright holder for this preprint (which was not certified by peer review) is the author/funder, who has granted medRxiv a license to display the preprint in perpetuity.

It is made available under a CC-BY-NC-ND 4.0 International license .

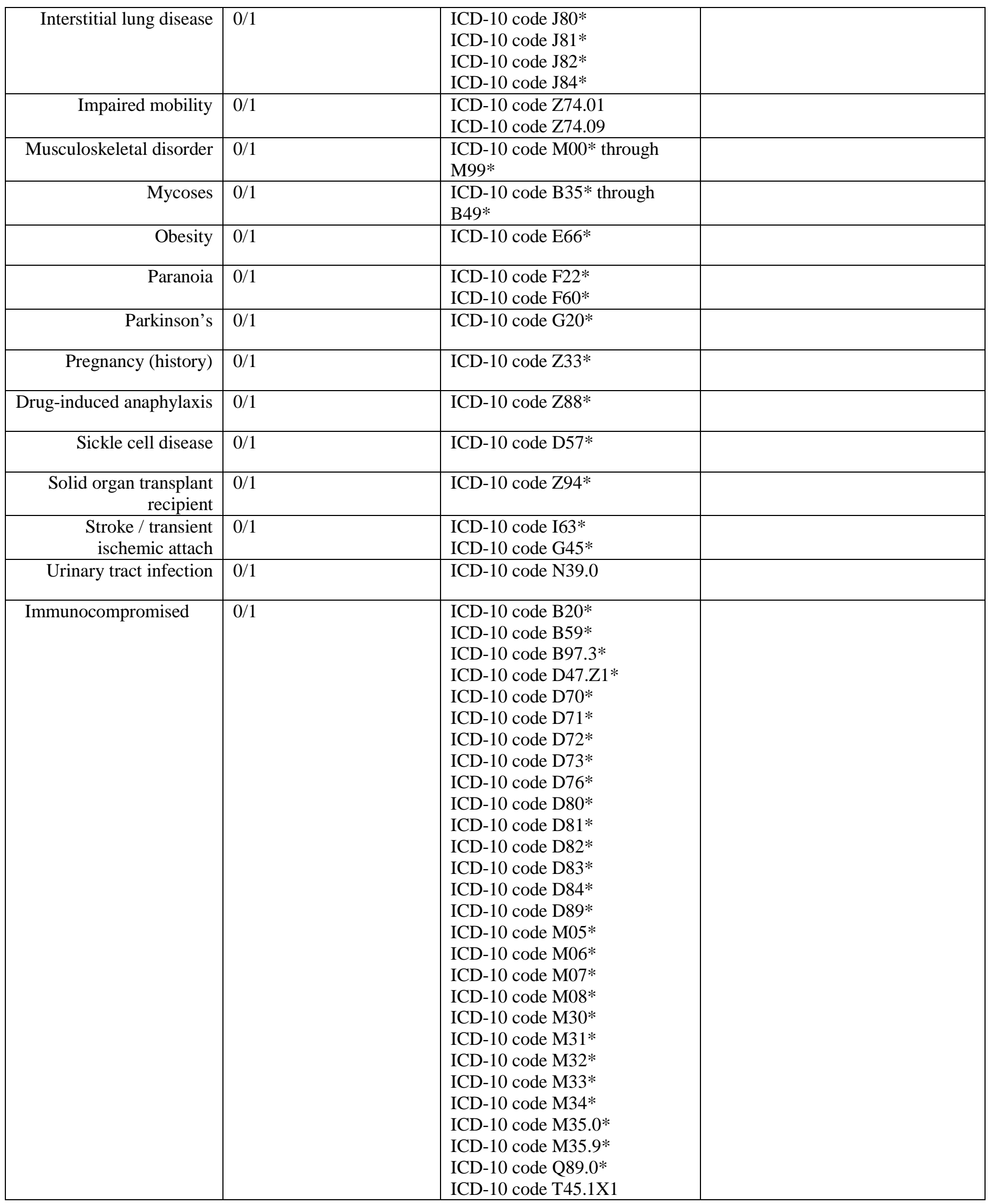


medRxiv preprint doi: https://doi.org/10.1101/2021.06.14.21258906; this version posted July 14, 2021. The copyright holder for this preprint (which was not certified by peer review) is the author/funder, who has granted medRxiv a license to display the preprint in perpetuity.

It is made available under a CC-BY-NC-ND 4.0 International license .

\begin{tabular}{|c|c|c|c|}
\hline & & $\begin{array}{l}\text { ICD-10 code } Z 21 * \\
\text { ICD-10 code } Z 48.2 * \\
\text { ICD-10 code } Z 51.0 * \\
\text { ICD-10 code } Z 51.1 * \\
\text { ICD-10 code } Z 94 *\end{array}$ & \\
\hline $\begin{array}{l}\text { Hematological } \\
\text { malignancy }\end{array}$ & $0 / 1$ & $\begin{array}{l}\text { ICD-10 codes } 81.70,81.79 \text {, } \\
81.90 \\
\text { ICD-10 codes } 82.90 \\
\text { ICD-10 codes } 84.10,84.40 \\
85.80 \\
\text { ICD-10 code C } 88.0 \\
\text { ICD-10 code C } 90.00 \\
\text { ICD-10 codes C91.00, 91.01, } \\
91.10,91.11,91.40 \\
\text { ICD-10 codes C92.00, 92.01, } \\
92.10,92.11,92.30 \\
96.2 \\
\text { ICD-10 code D47.2 }\end{array}$ & \\
\hline Vaccination status & $\begin{array}{l}\text { None } \\
\text { Partial } \\
\text { Full }\end{array}$ & $\begin{array}{l}\text { None: until first vaccination } \\
\text { date } \\
\text { Partial: from } 7 \text { days after the } \\
\text { first vaccination date and } \\
\text { before the second vaccination } \\
\text { date } \\
\text { Full: from } 7 \text { days after the } \\
\text { second vaccination date }\end{array}$ & $\begin{array}{l}\text { Determined at the specimen } \\
\text { collection date for SARS-CoV-2 } \\
\text { lab test during the study period } \\
\text { (December } 14,2020 \text { to March } 7 \text {, } \\
\text { 2021) }\end{array}$ \\
\hline Vaccine manufacturer & $\begin{array}{l}\text { None } \\
\text { Moderna } \\
\text { Pfizer }\end{array}$ & $\begin{array}{l}\text { None: no vaccination recorded } \\
\text { Moderna: CPT codes } 91301, \\
\text { 0011A, 0012A or vaccination } \\
\text { name } \\
\text { Pfizer: CPT codes } 91300, \\
\text { 0001A, 0002A or vaccination } \\
\text { name }\end{array}$ & $\begin{array}{l}\text { Determined at date of first } \\
\text { vaccination during the study period } \\
\text { (December 14, } 2020 \text { to March } 7 \text {, } \\
\text { 2021) }\end{array}$ \\
\hline $\begin{array}{r}\text { Month of SARS-CoV-2 } \\
\text { test }\end{array}$ & $\begin{array}{l}\text { 2020-Dec } \\
\text { 2021-Jan } \\
2021-\text { Feb } \\
\text { 2021-Mar }\end{array}$ & $\begin{array}{l}\text { Month of the specimen } \\
\text { collection date for SARS-CoV- } \\
2 \text { lab test }\end{array}$ & $\begin{array}{l}\text { Study period (December 14, } 2020 \\
\text { to March 7, 2021) }\end{array}$ \\
\hline $\begin{array}{r}\text { Care setting for SARS- } \\
\text { CoV-2 test }\end{array}$ & $\begin{array}{l}\text { ED/At Admission } \\
\text { Outpatient }\end{array}$ & $\begin{array}{l}\text { ED/At Admission: Specimen } \\
\text { collection date for SARS-CoV- } \\
2 \text { lab test on the same date as } \\
\text { ED visit or during an inpatient } \\
\text { stay } \\
\text { Outpatient: Specimen } \\
\text { collection date for SARS-CoV- } \\
2 \text { lab test on the same date as } \\
\text { outpatient or urgent care visit }\end{array}$ & $\begin{array}{l}\text { Assessed from specimen collection } \\
\text { dates and visit dates during the } \\
\text { study period (December 14, } 2020 \\
\text { to March 7, 2021) }\end{array}$ \\
\hline $\begin{array}{r}\text { Health and Human } \\
\text { Services (HHS) region }\end{array}$ & $\begin{array}{l}\text { Region } 1 \\
\text { Region } 2 \\
\text { Region } 3 \\
\text { Region } 4 \\
\text { Region } 5 \\
\text { Region } 6 \\
\text { Region } 7 \\
\text { Region } 8 \\
\text { Region } 9 \\
\text { Region } 10\end{array}$ & $\begin{array}{l}\text { Region 1: (state abbreviation) } \\
\text { CT, ME, NH, RI, VT } \\
\text { Region 2: NJ, NY } \\
\text { Region 3: DE, DC, MD, PA, } \\
\text { VA, WV } \\
\text { Region 4: AL, FL, GA, KY, } \\
\text { MS, NC, SC, TN } \\
\text { Region 5: IL, IN, MI, MN, OH, } \\
\text { WI } \\
\text { Region 6: AR, LA, NM, OK, }\end{array}$ & $\begin{array}{l}\text { Most recent available value based } \\
\text { on location of patient residency }\end{array}$ \\
\hline
\end{tabular}


medRxiv preprint doi: https://doi.org/10.1101/2021.06.14.21258906; this version posted July 14, 2021. The copyright holder for this preprint (which was not certified by peer review) is the author/funder, who has granted medRxiv a license to display the preprint in perpetuity.

It is made available under a CC-BY-NC-ND 4.0 International license .

\begin{tabular}{|c|c|c|c|}
\hline & & $\begin{array}{l}\text { TX } \\
\text { Region 7: IA, KS, MO, NE } \\
\text { Region 8: CO, MT, ND, SD, } \\
\text { UT, WY } \\
\text { Region 9: AZ, CA, HI, NV } \\
\text { Region 10: AK, ID, OR, WA }\end{array}$ & \\
\hline Homeless & $0 / 1$ & $\begin{array}{l}\text { 1= ICD-10 code Z59.0 or } \\
\text { Z59.1 } \\
\text { on any inpatient or outpatient } \\
\text { record }\end{array}$ & $\begin{array}{l}\text { During } 2 \text { years prior to specimen } \\
\text { collection date for SARS-CoV-2 } \\
\text { lab test }\end{array}$ \\
\hline Low income & $0 / 1$ & $\begin{array}{l}1=\text { income less than } 12,760, \\
\text { but not missing income } \\
0=\text { missing or income greater } \\
\text { than equal to } 12760\end{array}$ & $\begin{array}{l}\text { During } 2 \text { years prior to specimen } \\
\text { collection date for SARS-CoV-2 } \\
\text { lab test }\end{array}$ \\
\hline Quan-CCI & Numeric & $\begin{array}{l}\text { Using ICD-10 codes from any } \\
\text { inpatient or outpatient record, } \\
\text { computed Charlson } \\
\text { Comorbidity Index, Quan's } \\
\text { version [REF Quan H, } \\
\text { Sundararajan V, Halfon P, } \\
\text { Fong A, Burnand B, Luthi JC, } \\
\text { et al. Coding algorithms for } \\
\text { defining comorbidities in ICD- } \\
\text { 9-CM and ICD-10 } \\
\text { administrative data. Medical } \\
\text { Care 2005; 43(11):1130-1139. } \\
\text { DOI: } \\
\text { 10.1097/01.mlr.0000182534.19 } \\
\text { 832.83] }\end{array}$ & $\begin{array}{l}\text { During } 2 \text { years prior to and on date } \\
\text { of specimen collection date for } \\
\text { SARS-CoV-2 lab test }\end{array}$ \\
\hline BMI & $\begin{array}{l}\text { Normal } \\
\text { Overweight/ obese } \\
\text { Missing }\end{array}$ & $\begin{array}{l}\text { Normal: BMI less than } 26 \\
\text { Overweight/obese: BMI greater } \\
\text { than or equal to } 26\end{array}$ & $\begin{array}{l}\text { During } 2 \text { years prior to and on date } \\
\text { of specimen collection date for } \\
\text { SARS-CoV-2 lab test }\end{array}$ \\
\hline CAN Score & & $\begin{array}{l}\text { Score to identify patients at } \\
\text { highest risk of hospitalization } \\
\text { and mortality. As indicated in } \\
\text { VHA data. If missing, the score } \\
\text { is assumed to be } \\
\text { zero.[ https://www.va.gov/HEA } \\
\text { LTHCAREEXCELLENCE/abo } \\
\text { ut/organization/examples/care- } \\
\text { assessment-needs.asp] }\end{array}$ & $\begin{array}{l}\text { Most recent CAN score during } 2 \\
\text { years prior to and on date of } \\
\text { specimen collection date for } \\
\text { SARS-CoV-2 lab test }\end{array}$ \\
\hline Mortality-1 year & Percentage of probability & $\begin{array}{l}\text { Measurement of risk of } \\
\text { mortality in next year }\end{array}$ & \\
\hline Mortality-90 days & Percentage of probability & $\begin{array}{l}\text { Measurement of risk of } \\
\text { mortality in the next } 90 \text { days }\end{array}$ & \\
\hline Event-1 year & Percentage of probability & $\begin{array}{l}\text { Measurement of risk of any } \\
\text { visit or event in the next year }\end{array}$ & \\
\hline Event-90 days & Percentage of probability & $\begin{array}{l}\text { Measurement of risk of any } \\
\text { visit or event in the next } 90 \\
\text { days }\end{array}$ & \\
\hline Hospitalization-1 year & Percentage of probability & $\begin{array}{l}\text { Measurement of risk of a } \\
\text { hospitalization in the next year }\end{array}$ & \\
\hline Hospitalization-90 days & Percentage of probability & $\begin{array}{l}\text { Measurement of risk of a } \\
\text { hospitalization in the next } 90 \\
\text { days }\end{array}$ & \\
\hline
\end{tabular}


medRxiv preprint doi: https://doi.org/10.1101/2021.06.14.21258906; this version posted July 14, 2021. The copyright holder for this preprint (which was not certified by peer review) is the author/funder, who has granted medRxiv a license to display the preprint in perpetuity.

It is made available under a CC-BY-NC-ND 4.0 International license .

\begin{tabular}{|c|c|c|c|}
\hline COVID-19 symptoms & & $\begin{array}{l}\text { Symptoms as indicated from a } \\
\text { combination of text search on } \\
\text { inpatient admissions, ICD-10 } \\
\text { codes, or as indicated in the } \\
\text { COVID Domain }\end{array}$ & $\begin{array}{l}+/-14 \text { days around and including } \\
\text { the COVID-19 lab test specimen } \\
\text { date. }\end{array}$ \\
\hline Any & $0 / 1$ & Any one of the listed symptoms & \\
\hline None & $0 / 1$ & $\begin{array}{l}\text { Missing all of the listed } \\
\text { symptoms }\end{array}$ & \\
\hline Fever & $0 / 1$ & $\begin{array}{l}\text { ICD-10 code R50.9* } \\
\text { COVID domain variable } \\
\text { fever } 30 \mathrm{~d}\end{array}$ & \\
\hline Shortness of breath & $0 / 1$ & $\begin{array}{l}\text { ICD-10 code R06.02* } \\
\text { ICD-10 code R06.0* } \\
\text { ICD-10 code R09.02* } \\
\text { COVID domain variable } \\
\text { dyspnea30d }\end{array}$ & \\
\hline Cough & $0 / 1$ & $\begin{array}{l}\text { ICD-10 code R05* } \\
\text { COVID domain variable } \\
\text { cough } 30 \mathrm{~d}\end{array}$ & \\
\hline Loss of taste/smell & $0 / 1$ & $\begin{array}{l}\text { ICD-10 code } \mathrm{R} 43.9^{*} \\
\text { ICD-10 code } \mathrm{R} 43.8^{*} \\
\text { ICD-10 code } \mathrm{R} 43.1^{*} \\
\text { ICD-10 code } \mathrm{R} 43 * \\
\text { COVID domain variable } \\
\text { lossoftaste } 30 \mathrm{~d}\end{array}$ & \\
\hline Chills & $0 / 1$ & $\begin{array}{l}\text { ICD-10 code R68.83* } \\
\text { ICD-10 code R68.89* } \\
\text { COVID domain variable } \\
\text { chills30d }\end{array}$ & \\
\hline Diarrhea & $0 / 1$ & $\begin{array}{l}\text { ICD-10 code R19.7* } \\
\text { COVID domain variable } \\
\text { diarrhea30d }\end{array}$ & \\
\hline Sore throat & $0 / 1$ & $\begin{array}{l}\text { ICD-10 code J02.8* } \\
\text { ICD-10 code J02.9* } \\
\text { COVID domain variable } \\
\text { sorethroat } 30 \mathrm{~d}\end{array}$ & \\
\hline Myalgia & $0 / 1$ & $\begin{array}{l}\text { ICD-10 code M79.1* } \\
\text { COVID domain variable } \\
\text { myalgia30d }\end{array}$ & \\
\hline $\begin{array}{l}\text { Vaccination (other } \\
\text { pathogens) }\end{array}$ & & $\begin{array}{l}\text { Other pathogen vaccinations } \\
\text { identified by text search or CPT } \\
\text { code (listed below) }\end{array}$ & $\begin{array}{l}\text { The year prior to the specimen } \\
\text { collection date for SARS-CoV-2 } \\
\text { lab test }\end{array}$ \\
\hline $\begin{array}{r}\text { Influenza }(2019 / 20 \\
\text { season })\end{array}$ & $0 / 1$ & $\begin{array}{l}\text { CPT Code between } 90655 \text { and } \\
\text { 90659, Q2034 and Q2039 } \\
\text { CPT Code } 90662\end{array}$ & \\
\hline $\begin{array}{r}\text { Influenza }(2020 / 21 \\
\text { season })\end{array}$ & $0 / 1$ & $\begin{array}{l}\text { CPT Code between } 90655 \text { and } \\
\text { 90659, Q2034 and Q2039 } \\
\text { CPT Code } 90662\end{array}$ & \\
\hline
\end{tabular}


medRxiv preprint doi: https://doi.org/10.1101/2021.06.14.21258906; this version posted July 14, 2021. The copyright holder for this preprint (which was not certified by peer review) is the author/funder, who has granted medRxiv a license to display the preprint in perpetuity. It is made available under a CC-BY-NC-ND 4.0 International license .

Supplemental Table 2. Vaccine Effectiveness Against Laboratory Confirmed SARS-CoV-2 Infection Among Patients with COVID-19 Symptoms ${ }^{\mathrm{a}}$

\begin{tabular}{|c|c|c|c|c|}
\hline & \multicolumn{4}{|c|}{ VE, \% (95\%CI) } \\
\hline & \multicolumn{2}{|c|}{ Full vs. no vaccination } & \multicolumn{2}{|c|}{ Partial vs. no vaccination } \\
\hline & Unadjusted & Adjusted $^{\mathrm{b}}$ & Unadjusted & Adjusted $^{\mathrm{b}}$ \\
\hline Overall & $93(90,95)$ & $91(87,93)$ & $52(46,57)$ & $43(36,50)$ \\
\hline \multicolumn{5}{|c|}{ Age, years } \\
\hline $18-64$ & $90(77,96)$ & $89(73,95)$ & $40(22,54)$ & $31(10,48)$ \\
\hline$\geq 65$ & $89(84,92)$ & $88(83,91)$ & $43(35,49)$ & $42(33,49)$ \\
\hline $18-79$ & $90(85,93)$ & $89(85,93)$ & $42(33,49)$ & $40(31,47)$ \\
\hline$\geq 80$ & $88(77,93)$ & $88(79,94)$ & $44(29,56)$ & $38(21,52)$ \\
\hline \multicolumn{5}{|c|}{ VHA defined priority group } \\
\hline $1-4$ & $91(85,95)$ & $93(87,96)$ & $43(31,52)$ & $45(34,55)$ \\
\hline $5-6$ & $86(76,92)$ & $86(77,92)$ & $45(33,55)$ & $44(31,55)$ \\
\hline $7-8$ & $83(70,90)$ & $86(76,92)$ & $34(18,46)$ & $32(16,45)$ \\
\hline \multicolumn{5}{|c|}{ Race or ethnic group } \\
\hline Black & $94(85,97)$ & $94(85,98)$ & $31(13,45)$ & $31(13,45)$ \\
\hline Hispanic & $93(52,99)$ & $94(54,99)$ & $29(-21,58)$ & $16(-44,52)$ \\
\hline White & $87(81,91)$ & $86(80,90)$ & $48(40,55)$ & $43(34,51)$ \\
\hline Other & $96(68,99)$ & $93(49,99)$ & $47(12,68)$ & $37(-6,63)$ \\
\hline \multicolumn{5}{|c|}{ Sex } \\
\hline Female & $77(25,93)$ & $68(-6,90)$ & $59(21,79)$ & $41(-16,70)$ \\
\hline Male & $90(86,93)$ & $88(84,92)$ & $42(35,48)$ & $42(35,48)$ \\
\hline \multicolumn{5}{|c|}{ Urban-rural } \\
\hline Rural & $88(78,94)$ & $87(77,93)$ & $46(34,57)$ & $41(26,53)$ \\
\hline Urban & $90(86,93)$ & $89(84,92)$ & $40(31,48)$ & $41(32,49)$ \\
\hline \multicolumn{5}{|c|}{ BMI } \\
\hline Overweight/Obese & $82(66,90)$ & $83(68,91)$ & $31(9,47)$ & $39(20,54)$ \\
\hline \multicolumn{5}{|c|}{ Underlying medical conditions } \\
\hline Cancer & $79(58,89)$ & $77(56,88)$ & $37(13,55)$ & $37(11,55)$ \\
\hline Congestive heart failure & $77(60,87)$ & $76(58,87)$ & $43(24,57)$ & $42(23,57)$ \\
\hline Chronic kidney disease & $88(79,94)$ & $88(78,94)$ & $40(23,53)$ & $43(27,56)$ \\
\hline Diabetes mellitus & $91(86,95)$ & $92(86,95)$ & $47(37,55)$ & $45(34,54)$ \\
\hline Hypertension & $88(83,92)$ & $87(82,91)$ & $47(38,54)$ & $42(32,50)$ \\
\hline Immuno-compromised & $82(71,88)$ & $83(72,89)$ & $28(11,42)$ & $34(18,47)$ \\
\hline
\end{tabular}


medRxiv preprint doi: https://doi.org/10.1101/2021.06.14.21258906; this version posted July 14, 2021. The copyright holder for this preprint (which was not certified by peer review) is the author/funder, who has granted medRxiv a license to display the preprint in perpetuity.

It is made available under a CC-BY-NC-ND 4.0 International license.

Supplemental Table 3. Vaccination Status for Cases and Controls in Analysis of Vaccine Effectiveness Against COVID-19-related Hospitalization and Death

\begin{tabular}{|c|c|c|c|c|}
\hline \multicolumn{5}{|c|}{ COVID-19-related hospitalization } \\
\hline & \multicolumn{2}{|c|}{ Unmatched } & \multicolumn{2}{|c|}{ Matched } \\
\hline & $\begin{array}{c}\text { Cases } \\
(\mathrm{n}=3,209)\end{array}$ & $\begin{array}{c}\text { Controls } \\
(\mathrm{n}=316,895)\end{array}$ & $\begin{array}{c}\text { Cases } \\
(\mathrm{n}=3,209)\end{array}$ & $\begin{array}{c}\text { Controls } \\
(\mathrm{n}=13,075)\end{array}$ \\
\hline \multicolumn{5}{|c|}{ COVID-19 vaccination status } \\
\hline Full & 17 & 11,656 & 17 & 442 \\
\hline Partial & 151 & 18,901 & 151 & 843 \\
\hline None & 3,041 & 286,338 & 3,041 & 11,790 \\
\hline \multicolumn{5}{|c|}{ COVID-19-related death } \\
\hline & \multicolumn{2}{|c|}{ Unmatched } & \multicolumn{2}{|c|}{ Matched } \\
\hline & $\begin{array}{l}\text { Cases } \\
(\mathrm{n}=669)\end{array}$ & $\begin{array}{c}\text { Controls } \\
(n=316,895)\end{array}$ & $\begin{array}{c}\text { Cases } \\
(\mathrm{n}=669)\end{array}$ & $\begin{array}{c}\text { Controls } \\
(\mathrm{n}=2,728)\end{array}$ \\
\hline \multicolumn{5}{|c|}{ COVID-19 vaccination status } \\
\hline Vaccinated $*$ & 25 & 30,557 & 25 & 215 \\
\hline None & 644 & 286,338 & 644 & 2,513 \\
\hline
\end{tabular}


medRxiv preprint doi: https://doi.org/10.1101/2021.06.14.21258906; this version posted July 14, 2021. The copyright holder for this preprint (which was not certified by peer review) is the author/funder, who has granted medRxiv a license to display the preprint in perpetuity. It is made available under a CC-BY-NC-ND 4.0 International license .

Supplemental Table 4. Vaccine Effectiveness Against Laboratory Confirmed SARS-CoV-2 Infection, Combining VA and CMS Data ${ }^{\mathrm{a}, \mathrm{b}}$

\begin{tabular}{|c|c|c|c|c|}
\hline & \multicolumn{4}{|c|}{ VE, \% (95\%CI) } \\
\hline & \multicolumn{2}{|c|}{ Full vs. no vaccination } & \multicolumn{2}{|c|}{ Partial vs. no vaccination } \\
\hline & Unadjusted & Adjusted $^{\mathrm{c}}$ & Unadjusted & Adjusted $^{\mathrm{c}}$ \\
\hline Overall & $87(83,89)$ & $87(83,89)$ & $43(37,48)$ & $42(36,48)$ \\
\hline \multicolumn{5}{|c|}{ Age, $y$} \\
\hline $18-64$ & $89(77,95)$ & $90(79,95)$ & $44(27,57)$ & $43(26,57)$ \\
\hline$\geq 65$ & $88(84,91)$ & $88(84,91)$ & $47(41,53)$ & $46(39,51)$ \\
\hline \multicolumn{5}{|c|}{ VHA defined priority group } \\
\hline $1-4$ & $87(79,92)$ & $87(80,92)$ & $52(42,61)$ & $51(40,60)$ \\
\hline $5-6$ & $84(72,90)$ & $83(71,90)$ & $44(30,56)$ & $45(30,56)$ \\
\hline $7-8$ & $85(69,93)$ & $83(64,92)$ & $22(-3,41)$ & $24(-2,43)$ \\
\hline \multicolumn{5}{|c|}{ Race or ethnic group } \\
\hline Black & $92(82,96)$ & $91(81,96)$ & $37(16,52)$ & $30(7,47)$ \\
\hline White & $83(78,88)$ & $84(78,88)$ & $44(36,51)$ & $46(39,53)$ \\
\hline \multicolumn{5}{|c|}{ Sex } \\
\hline Male & $87(83,90)$ & $87(83,90)$ & $42(36,48)$ & $41(34,46)$ \\
\hline \multicolumn{5}{|c|}{ Urban-rural } \\
\hline Rural & $87(76,93)$ & $87(76,93)$ & $45(30,57)$ & $46(31,58)$ \\
\hline Urban & $84(78,88)$ & $83(78,88)$ & $38(30,45)$ & $36(27,44)$ \\
\hline \multicolumn{5}{|c|}{ BMI } \\
\hline Obese & $84(75,90)$ & $83(74,89)$ & $29(15,40)$ & $27(12,39)$ \\
\hline \multicolumn{5}{|c|}{ Underlying medical conditions } \\
\hline Congestive heart failure & $67(10,88)$ & $71(17,90)$ & $47(8,70)$ & $49(9,72)$ \\
\hline Chronic kidney disease & $86(53,96)$ & $87(56,96)$ & $51(23,69)$ & $47(15,67)$ \\
\hline Diabetes mellitus & $90(81,94)$ & $89(80,94)$ & $36(20,49)$ & $34(17,48)$ \\
\hline Hypertension & $85(78,89)$ & $85(79,90)$ & $35(24,45)$ & $36(24,45)$ \\
\hline Immunocompromised & $65(32,82)$ & $67(35,83)$ & $33(6,52)$ & $37(11,55)$ \\
\hline \multicolumn{5}{|c|}{$\begin{array}{l}\text { Abbreviations: BMI, body mass index; CMS, Centers for Medicare and Medicaid Services; ICD-10, } \\
\text { International Classification of Diseases } 10^{\text {th }} \text { Revision; VA, Veterans Affairs; VE, vaccine effectiveness. } \\
\text { "Since 2020, for VHA enrolled patients with Medicare who have either tested positive for SARS-Cov-2 at } \\
\text { a VHA facility or who have a COVID-19 medical claim in Medicare (ICD-10 codes B97.29 ["Other } \\
\text { coronavirus"] and U07.1 ["COVID-19"] diagnosis code in inpatient, skilled nursing facility, institutional } \\
\text { outpatient, hospice, and carrier/Part B files), the VHA receives the patients' Medicare data with a 4-5 } \\
\text { week lag time [REF https://vaww.virec.research.va.gov/VACMS/Medicare/COVID19-Data.htm]. } \\
\text { 'See Supplemental Table } 1 \text { for definitions of variables in this table. } \\
\text { cThe adjusted variables include the following: age, BMI, cancer, congestive heart failure, chronic kidney } \\
\text { disease, diabetes mellitus, hypertension, immunocompromised, VA priority level, race, sex, rurality. }\end{array}$} \\
\hline
\end{tabular}


Supplemental Table 5. Vaccine Effectiveness Against Laboratory Confirmed SARS-CoV-2 Infection by Type of Diagnostic Test ${ }^{\mathrm{a}}$

\begin{tabular}{|l|c|c|c|c|}
\hline & \multicolumn{4}{|c|}{ VE, \% (95\%CI) } \\
\hline Overall & \multicolumn{2}{|c|}{ Full vs. no vaccination } & \multicolumn{2}{c|}{ Partial vs. no vaccination } \\
\hline & Unadjusted & Adjusted $^{\mathrm{b}}$ & Unadjusted & Adjusted $^{\mathrm{b}}$ \\
\hline PCR test & $95(93,96)$ & $92(89,94)$ & $68(64,70)$ & $53(49,58)$ \\
\hline Antigen test & $96(90,99)$ & $97(90,99)$ & $54(33,69)$ & $62(42,75)$ \\
\hline
\end{tabular}

Abbreviations: BMI, body mass index; VE, vaccine effectiveness; VHA, Veterans Health Affairs. ${ }^{\mathrm{a}}$ See Supplemental Table 1 for definitions of variables in this table.

bThe adjusted variables include the following: age, BMI, cancer, congestive heart failure, chronic kidney disease, diabetes mellitus, hypertension, immunocompromised, VA priority level, race, sex, rurality. 
medRxiv preprint doi: https://doi.org/10.1101/2021.06.14.21258906; this version posted July 14, 2021. The copyright holder for this preprint (which was not certified by peer review) is the author/funder, who has granted medRxiv a license to display the preprint in perpetuity. It is made available under a CC-BY-NC-ND 4.0 International license .

References

1. FDA. FDA Takes Key Action in Fight Against COVID-19 By Issuing Emergency Use Authorization for First COVID-19 Vaccine [Available from: https://www.fda.gov/newsevents/press-announcements/fda-takes-key-action-fight-against-covid-19-issuing-emergencyuse-authorization-first-covid-19.

2. FDA. FDA Takes Additional Action in Fight Against COVID-19 By Issuing Emergency Use Authorization for Second COVID-19 Vaccine [Available from: https://www.fda.gov/newsevents/press-announcements/fda-takes-additional-action-fight-against-covid-19-issuingemergency-use-authorization-second-covid.

3. Affairs DoV. Department of Veterans Affairs COVID-19 National Summary [Available from: https://www.accesstocare.va.gov/Healthcare/COVID19NationalSummary.

4. Affairs TUSSCoV. BREAKING: Bipartisan Bill Expanding Vaccine Access for Vets \& Families Signed Into Law [Available from:

https://www.veterans.senate.gov/newsroom/majority-news/breaking-bipartisan-bill-expandingvaccine-access-for-vets-and-families-signed-into-law.

5. Polack FP, Thomas SJ, Kitchin N, Absalon J, Gurtman A, Lockhart S, et al. Safety and Efficacy of the BNT162b2 mRNA Covid-19 Vaccine. N Engl J Med. 2020;383(27):2603-15.

6. Baden LR, El Sahly HM, Essink B, Kotloff K, Frey S, Novak R, et al. Efficacy and Safety of the mRNA-1273 SARS-CoV-2 Vaccine. N Engl J Med. 2021;384(5):403-16.

7. Haas EJ, Angulo FJ, McLaughlin JM, Anis E, Singer SR, Khan F, et al. Impact and effectiveness of mRNA BNT162b2 vaccine against SARS-CoV-2 infections and COVID-19 cases, hospitalisations, and deaths following a nationwide vaccination campaign in Israel: an observational study using national surveillance data. Lancet. 2021;397(10287):1819-29.

8. Dagan N, Barda N, Kepten E, Miron O, Perchik S, Katz MA, et al. BNT162b2 mRNA Covid-19 Vaccine in a Nationwide Mass Vaccination Setting. N Engl J Med. 2021;384(15):1412-23.

9. Hall VJ, Foulkes S, Saei A, Andrews N, Oguti B, Charlett A, et al. COVID-19 vaccine coverage in health-care workers in England and effectiveness of BNT162b2 mRNA vaccine against infection (SIREN): a prospective, multicentre, cohort study. Lancet. 2021;397(10286):1725-35.

10. Thompson MG, Burgess JL, Naleway AL, Tyner HL, Yoon SK, Meece J, et al. Interim Estimates of Vaccine Effectiveness of BNT162b2 and mRNA-1273 COVID-19 Vaccines in Preventing SARS-CoV-2 Infection Among Health Care Personnel, First Responders, and Other Essential and Frontline Workers - Eight U.S. Locations, December 2020-March 2021. MMWR Morb Mortal Wkly Rep. 2021;70(13):495-500.

11. Tenforde MW, Olson SM, Self WH, Talbot HK, Lindsell CJ, Steingrub JS, et al. Effectiveness of Pfizer-BioNTech and Moderna Vaccines Against COVID-19 Among Hospitalized Adults Aged >/=65 Years - United States, January-March 2021. MMWR Morb Mortal Wkly Rep. 2021;70(18):674-9.

12. Lopez Bernal J, Andrews N, Gower C, Robertson C, Stowe J, Tessier E, et al. Effectiveness of the Pfizer-BioNTech and Oxford-AstraZeneca vaccines on covid-19 related symptoms, hospital admissions, and mortality in older adults in England: test negative casecontrol study. Bmj. 2021;373:n1088. 
medRxiv preprint doi: https://doi.org/10.1101/2021.06.14.21258906; this version posted July 14, 2021. The copyright holder for this preprint (which was not certified by peer review) is the author/funder, who has granted medRxiv a license to display the preprint in perpetuity. It is made available under a CC-BY-NC-ND 4.0 International license .

13. Angel Y, Spitzer A, Henig O, Saiag E, Sprecher E, Padova H, et al. Association Between Vaccination With BNT162b2 and Incidence of Symptomatic and Asymptomatic SARS-CoV-2 Infections Among Health Care Workers. JAMA. 2021.

14. Fung M, Babik JM. COVID-19 in Immunocompromised Hosts: What We Know So Far. Clin Infect Dis. 2021;72(2):340-50.

15. Lee LYW, Cazier JB, Starkey T, Briggs SEW, Arnold R, Bisht V, et al. COVID-19 prevalence and mortality in patients with cancer and the effect of primary tumour subtype and patient demographics: a prospective cohort study. Lancet Oncol. 2020;21(10):1309-16.

16. Vahidy FS, Nicolas JC, Meeks JR, Khan O, Pan A, Jones SL, et al. Racial and ethnic disparities in SARS-CoV-2 pandemic: analysis of a COVID-19 observational registry for a diverse US metropolitan population. BMJ Open. 2020;10(8):e039849.

17. Rentsch CT, Kidwai-Khan F, Tate JP, Park LS, King JT, Jr., Skanderson M, et al. Patterns of COVID-19 testing and mortality by race and ethnicity among United States veterans: A nationwide cohort study. PLoS Med. 2020;17(9):e1003379.

18. Abedi V, Olulana O, Avula V, Chaudhary D, Khan A, Shahjouei S, et al. Racial, Economic and Health Inequality and COVID-19 Infection in the United States. medRxiv. 2020.

19. CDC. COVID Data Tracker [Available from: https://covid.cdc.gov/covid-datatracker/\#vaccination-demographics-trends]. .

20. Patel MM, Jackson ML, Ferdinands J. Postlicensure Evaluation of COVID-19 Vaccines. JAMA. 2020;324(19):1939-40.

21. McDermott D, Hudman, J., Cox, C. The Veteran Health Administration's Role During the COVID-19 Response [Available from: https://www.healthsystemtracker.org/brief/theveteran-health-administrations-role-during-the-covid-19-response/. 